Revista Brasileira de Cartografia

ISSN 1808-0936 | https://doi.org/10.14393/revbrascartogr

Sociedade Brasileira de Cartografia, Geodésia, Fotogrametria e Sensoriamento Remoto

\title{
Determinação de Velocidades das Estações da RBMC com Uso do Software SARI
}

\section{Velocities Determination of RBMC Stations Using the SARI Software}

\author{
Marcony de Paulo Ramos ${ }^{1}$, William Rodrigo Dal Poz ${ }^{2}$ e Alessandro Salles Carvalho ${ }^{3}$
}

1 Universidade Federal da Bahia - UFBA, Departamento de Engenharia de Transportes e Geodesia, Salvador, Brasil e Universidade Federal de Viçosa - UFV, Departamento de Engenharia Civil, Viçosa-MG, Brasil. E-mail. marcony.paulo@yahoo.com.br. ORCID: http://orcid.org/0000-0002-7423-7727

2 Universidade Federal de Viçosa, Departamento de Engenharia Civil, Viçosa-MG, Viçosa, Brasil. E-mail. william.dalpoz@ufv.br. ORCID: https://orcid.org/0000-0001-9532-3643

3 Universidade Federal de Juiz de Fora, Faculdade de Engenharia - Departamento de Transportes e Geotecnia, Juiz de Fora, Brasil. E-mail. alessandro.carvalho@ufjf.edu.br.

ORCID: https://orcid.org/0000-0001-7428-0912

Recebido: 06.2020 | Aceito: 10.2020

Resumo: Devido ao aperfeiçoamento da precisão do posicionamento com GNSS (Global Navigation Satellite System) e à instalação de grandes redes de operação contínua, longas séries temporais de coordenadas do GNSS agora são amplamente utilizadas para conhecer uma variedade de processos de deformação da Terra. Logo, uma tarefa que passou a ser muito utilizada é a determinação de velocidades de estações das redes de monitoramento contínuo, tendo diversas aplicações na geodésia. As componentes de velocidades de uma estação podem ser determinadas de modo simplificado, como resultado de uma regressão linear simples, assumindo que o comportamento da velocidade de uma estação responde a um modelo linear. Porém, para obter precisão melhor, é importante considerar que a velocidade da estação não esteja relacionada apenas à tendência, mas também em função da sazonalidade e de ruídos. É comum pressupor a presença de ruídos brancos em uma série temporal de coordenadas GNSS, porém, é importante estudar ruídos da lei de potência para determinar o tipo de ruído em cada série. Dessa forma, este trabalho teve como objetivo realizar a determinação de velocidades das estações considerando tendência, sazonalidade e tipos de ruídos presentes nas séries. Os estudos foram realizados a partir de 94 estações pertencentes à RBMC (Rede Brasileira de Monitoramento Contínuo dos Sistemas GNSS), pós-processadas no serviço online IBGE-PPP (Instituto Brasileiro de Geografia e Estatísticas - Posicionamento por Ponto Preciso) e análises das séries de coordenadas executada no software SARI (Señales y Análisis de Ruido Interactivo). Os resultados mostram que não há diferenças significativas na obtenção das velocidades planimétricas das estações quando é negligenciada a sazonalidade, bem como mostram que as análises de ruídos não afetam as velocidades das estações analisadas nesta pesquisa.

Palavras-chave: GNSS. SARI. Séries temporais. Modelo de velocidade.

\begin{abstract}
Lengthy GNSS (Global Navigation Satellite System) coordinate time series are now widely used to assess a variety of Earth deformation processes due to the improvement of GNSS accuracy and the installation of large networks of continuous operation. Therefore, a task that has come to be widely used is the determination of station velocity of the continuous monitoring networks, having several applications in geodesy. The velocity components of a station can be determined in a simplified way, as a result of a simple linear regression, assuming that the behavior of a station's velocity responds to a linear model. However, to obtain more precision, it is critical to consider that the velocity of the season is not only related to the trend, but also as a function of seasonality and noise. It is common to assume the presence of white noise in a time series of GNSS coordinates, however, it is important to study power-law noise to determine the type of noise in each series. Thus, this study aimed to determine the velocity of stations considering trends, seasonality, and types of noise present in the series. The studies were carried out from 94 stations belonging to RBMC (Brazilian Network for Continuous Monitoring of the GNSS Systems), post-processed in the IBGE-PPP (Brazilian Institute of Geography and Statistics - Precise Point Positioning) online service and analyzes of the series of coordinates performed in the SARI (Señales y Análisis de Ruido Interactivo) software. The results indicate no significant differences in obtaining the planimetric velocity of the stations when seasonality is neglected. The research also conveys that the noise analysis does not affect the velocities of the stations analyzed.
\end{abstract}

Keywords: GNSS. SARI. Time Series. Velocity Model. 


\section{INTRODUÇÃO}

As velocidades das estações GNSS (Global Navigation Satellite Systems) são obtidas por meio da análise de séries temporais de coordenadas GNSS. Estas séries temporais contêm sinais induzidos pela deformação da Terra, mas também por erros sistemáticos (tendência), em diferentes escalas de tempo, desde a deformação das marés sub-diárias até a deformação interanual de carregamento de superfície e rotação da placa tectônica secular (SANTAMARÍA-GÓMEZ, 2019).

Segundo Silva e Costa (2008), a metodologia aplicada na determinação do deslocamento horizontal de cada estação baseia-se na condição de linearidade do movimento das placas tectônicas. Essa condição também é utilizada em modelos geofísicos de movimento de placas. Segundo Zamora (2014), as componentes de velocidades de uma estação podem ser determinadas relativamente de forma fácil, como resultado de uma regressão linear simples, assumindo que o comportamento da velocidade de uma estação responde a um modelo linear. $\mathrm{O}$ autor ressalta que é necessário obter o vetor posição em função do tempo (séries temporais de coordenadas do GNSS) para realizar o ajuste pelo método dos mínimos quadrados (MMQ).

Alguns autores consideram que a velocidade da estação não está relacionada apenas à tendência (modelo linear), mas também com a sazonalidade e ruídos (HE et al., 2017; CHOUSIANITIS et al., 2013; KLOS; BOS; BOGUSZ, 2018; BOCK et al., 2016; BOGUSZ et al., 2016; LUNA et al., 2017; NIKOLAIDIS, 2002). Salienta-se que grande parte destes trabalhos teve por finalidade a determinação dos tipos de ruídos e sazonalidade presentes no movimento da estação, em que é necessária uma análise mais detalhada de todos os fatores que afetam as séries temporais de coordenadas do GNSS.

Segundo He et al., 2019, o modelo mais comum para descrever as propriedades estocásticas dos ruídos nas séries temporais de coordenadas do GNSS é a combinação entre os ruídos de cintilação e branco. Já Berthier et al., 2019 estudaram, a partir de séries temporais de coordenadas do GNSS, os efeitos da sazonalidade na determinação de velocidade vertical de estação GNSS para analisar a deformação induzida por carga sazonal.

Vários são os trabalhos realizados com intuito de determinar a tendência das séries temporais com maior acurácia (BENOIST et al., 2020; WILLIAMS et al., 2004; SANTAMARÍA-GÓMEZ et al., 2011; BOS et al., 2020). Neste ínterim, Santamaría-Gómez (2019) implementou o software SARI (Señales y Análisis de Ruido Interactivo) para realizar o processamento de séries temporais de dados GNSS e permitir aos usuários visualizarem séries temporais de posições GNSS, removerem interativamente outliers e descontinuidades, bem como ajustar modelos e salvar os resultados. Mazzotti et al. (2020) realizaram diversas análises em séries temporais de coordenadas do GNSS com intuito de verificar a eficiência de diversos softwares na determinação de velocidade de estações, sendo o software SARI aquele que obteve resultados mais satisfatórios.

Para que seja possível obter as séries de coordenadas obtidas com GNSS são necessárias estações GNSS de monitoramento contínuo. Dessa forma, serão utilizados dados GNSS da RBMC (Rede Brasileira de Monitoramento Contínuo dos Sistemas GNSS), que possui todas as estações pertencentes à placa tectônica Sul-Americana, localizada em território brasileiro, a qual é alvo de estudo para esta pesquisa. Diversos trabalhos foram realizados com intuito de determinação de velocidades de estações presentes nessa placa, mas poucos realizaram análise de ruído e sazonalidade nas séries temporais de coordenadas obtidas com GNSS (SOUZA, 2013; PEREZ, 2002; PEREZ; MONICO; CHAVES, 2003; SÁNCHEZ; DREWES, 2016; SALAMANCA, 2016; CARVALHO, 2015; COSTA, 2001; SILVA; COSTA, 2008). Quando não é possível realizar a determinação de velocidade de estações GNSS pode-se utilizar modelo de velocidade, o SIRGAS (Sistema de Referência Geocêntrico para as Américas) atualiza continuamente, desde 2003, o VEMOS (Velocity Model of SIRGAS). Já foram lançando quatro versões do VEMOS que são: VEMOS2003(referenciado ao ITRF2000 - International Terrestrial Reference Frame 2000); VEMOS2009 (referenciado ao ITRF2015); VEMOS2015(referenciado ao ITRF2008); e o mais atual sendo o VEMOS2017 (referenciado ao ITRF2014). O VEMOS2017 que foi derivado de velocidades pontuais de 515 estações geodésicas de 01/01/2014 a 28/01/2017. De acordo com Drewes e Sánchez (2020), os melhores valores de incerteza acontecem na região mais estável da placa Sul-Americana.

Portanto, este trabalho teve como propósito determinar as velocidades de 94 estações pertencentes à RBMC (processadas no serviço online IBGE-PPP - Instituto Brasileiro de Geografia e Estatísticas - 
Posicionamento por Ponto Preciso) a partir de séries temporais com análise de sazonalidades e ruídos no software SARI.

\section{SÉRIES TEMPORAIS DE COORDENADAS GNSS}

Uma série temporal pode ser definida como uma sequência de observações tomadas no tempo ou qualquer conjunto de dados ou variáveis ordenadas no tempo (MORETTIN; TOLOI, 2006). De acordo com Box, Jenkins e Reinsel (2008), uma das principais peculiaridades no desenvolvimento de modelos de séries temporais é a suposição de alguma forma de equilíbrio estatístico. Um pressuposto particular desse tipo é o da estacionariedade. Geralmente, uma série temporal estacionária pode ser descrita de maneira útil por sua média, variância e função de autocorrelação ou por sua variação média e função de densidade espectral.

Os efeitos presentes nas séries temporais podem ser removidos mediante o ajustamento de uma função adequada aos dados. O vetor de resíduos do processo de ajustamento corresponde aos efeitos considerados no modelo matemático da série filtrada. A escolha do modelo matemático apropriado geralmente é feita com base no conhecimento de algumas hipóteses, ou seja, considera-se que a série esteja isenta de deslocamento, tendência e efeitos de natureza cíclica (NIKOLAIDIS, 2002).

A utilização e análise de séries temporais por pesquisadores de geociências vêm sendo cada vez mais empregadas como, por exemplo: no monitoramento de deformação da crosta; na eliminação de efeitos sazonais de coordenadas GPS (Global Positioning System); no monitoramento de ruídos em dados de monitoramento de barragens; na análise de multicaminho dos sinais GNSS em estações de monitoramento contínuo; na verificação de influência na carga hidrológica na altitude geométrica; na estimativa de tendências e na determinação de velocidade de estações (GEIRSSON, 2003; ROSA, 2008; LIMA, 2012; ALVES et al., 2013; NASCIMENTO, 2016; DIDOVA et al., 2016; ALINIA; TIAPO; JAMES, 2017).

A análise da série temporal possibilita extrair características de eventos que ocorrem no decorrer do tempo, tornando possível identificar os fatores que, de alguma maneira, regem o comportamento delas (ROSA, 2008). Isto evidencia a potencialidade das análises de séries temporais no tocante às técnicas para a verificação de tendência, sazonalidade e ruído (CAÑADA, 2016). A tendência é definida como um padrão de crescimento ou decrescimento da função em certo período de tempo. A tendência nas séries temporais de coordenadas do GNSS corresponde à velocidade da estação.

A sazonalidade representa o comportamento periódico da série temporal, ou seja, quando as similaridades na série ocorrem após intervalos de tempo básicos. A sazonalidade pode ser estimada mediante o ajuste de uma função que melhor descreva o comportamento da série. De modo geral, uma série apresenta comportamento cíclico onde os métodos empregados procuram encontrar um modelo que a represente. Desta forma, o ajuste de uma função trigonométrica apontaria características importantes, como amplitude e frequência dos ciclos (MORETTIN; TOLOI, 2006). Para realizar o ajuste da série, algumas técnicas podem ser utilizadas, como: filtro de Kalmam, wavelet (ondeletas), transformação de Fourier e o MMQ, além da integração entre alguns dos métodos supracitados (ROSA, 2008; SANTAMARÍA-GÓMEZ, 2019).

Com relação ao ruído, é comum pressupor a presença apenas de ruídos brancos em uma série temporal de coordenadas GNSS, com objetivo de simplificar as análises. Porém, vários estudos são realizados com objetivo de obter as tendências das séries temporais com precisão melhor. O estudo do espectro de potência (k) é usado para determinar o tipo de ruído em cada série. Assim, o modelo de ruído de lei de potência é ruído branco (white) quando $\mathrm{k}$ igual à zero $(\mathrm{k}=0)$; o ruído de cintilação (flicker) quando $\mathrm{k}$ igual a -1 , e $\mathrm{k}=-2$ é ruído de passeio aleatório (random walk) (WANG; HERRING, 2019).

Segundo Klos et al. (2018), o ruído começa a se tornar mais importante que os sinais periódicos (sazonalidade) para séries temporais com mais de 9 anos. Ou seja, os sinais periódicos são mais importantes para escalas de tempo curtas, enquanto o ruído estocástico desempenha um papel significativo quando a duração da série temporal aumenta.

O estudo do espectro de potência $(\mathrm{k})$ tem sido usado para determinar o tipo de ruído em cada série. Vários estudos determinam a presença de ruído correlacionado com o tempo e seu efeito na estimativa da incerteza da série. Segundo Santamaría-Gómez (2019), o modelo de análise de ruído com lei de potência e o 
ruído branco são as que melhor descrevem as características de ruído nas três componentes de séries temporais de coordenadas do GNSS.

Conforme Caporali (2003), a densidade espectral de potência das séries temporais é usada para inferir sobre a variação da mudança na inclinação (tendência) e identificar, com base no conhecimento dos índices espectrais, o tipo de ruído presente na série. Portanto, é possível considerar os ruídos na modelagem matemática utilizada na determinação das velocidades e, deste modo, melhorar a precisão desta estimativa. A partir dessas séries temporais ajustadas (após as análises de tendência, sazonalidade ruídos), é possível obter as velocidades das estações.

\subsection{Análise de séries temporais no SARI}

O SARI foi desenvolvido na linguagem de programação R sob a estrutura interativa do pacote Shiny R. Ele representa um conjunto de ferramentas de análise interativas de séries temporais de coordenadas obtidas com GNSS que foi criado com foco na visualização em série e processamento de sinais usando MMQ, filtro de Kalman e análise de ruído estocástico do MLE (Maximum-Likelihood Estimation) (SANTAMARÍAGÓMEZ, 2019).

O software pode ser utilizado em um computador local ou remotamente a partir de um servidor público da Web acessível em https://alvarosg.shinyapps.io/sari/. A vantagem da última opção é que o usuário não precisa se preocupar com os próprios recursos de computação. O SARI utiliza uma interface interativa baseada em navegador para visualizar séries de dados, ajustar modelos multiparâmetros e analisar os resíduos (SANTAMARÍA-GÓMEZ, 2019).

De acordo com Santamaría-Gómez (2019), o software está orientado para séries temporais diárias e semanais de posição GNSS, porém, qualquer outra série de dados pode ser analisada, desde que seja utilizado o formato tabulado consistente. O SARI disponibiliza dois métodos de ajustamento: o método dos mínimos quadrados (MMQ) e filtro de Kalman.

O ajustamento pelo MMQ usa o algoritmo clássico de Gauss-Newton. Santamaría-Gómez (2019) afirma que, a precisão dos parâmetros estimados no MMQ é fornecida assumindo que os resíduos são aleatórios e independentes, possuindo distribuição normal. Salienta-se que as duas últimas hipóteses não são geralmente satisfeitas quando ocorre a presença de ruído relacionado ao tempo na série. Já o método de ajustamento pelo filtro de Kalman foi implementado em dois tipos diferentes: o EKF (Extended Kalman Filter) e o UKF (Unscented Kalman Filter). Segundo Santamaría-Gómez (2019), ambas as implementações de filtro Kalman são baseadas no algoritmo RTS (Rauch Tung Striebel) (RAUCH; TUNG; STRIEBEL, 1965).

O software tem cinco abas de ferramentas principais, que são:

a) Dados e formato de entrada: entrada dos arquivos, definição dos formatos e redução da amostragem da série.

b) Controles de plotagem: plota a série e realiza a interação para remoção de outliers.

c) Informações auxiliares: entrada de arquivos auxiliares relativos às séries temporais. Por exemplo, uma série secundária ou um arquivo de descontinuidade.

d) Controles de ajuste: métodos de ajustamento da série e os componentes do modelo.

e) Ajuste adicional: ferramentas importantes no ajustamento de séries temporais.

A Figura 1 ilustra as principais ferramentas do software SARI. Salienta-se que a figura não corresponde ao formato real do SARI; apenas foi organizado com esta configuração para um melhor entendimento. 
Figura 1 - Principais ferramentas do software SARI.

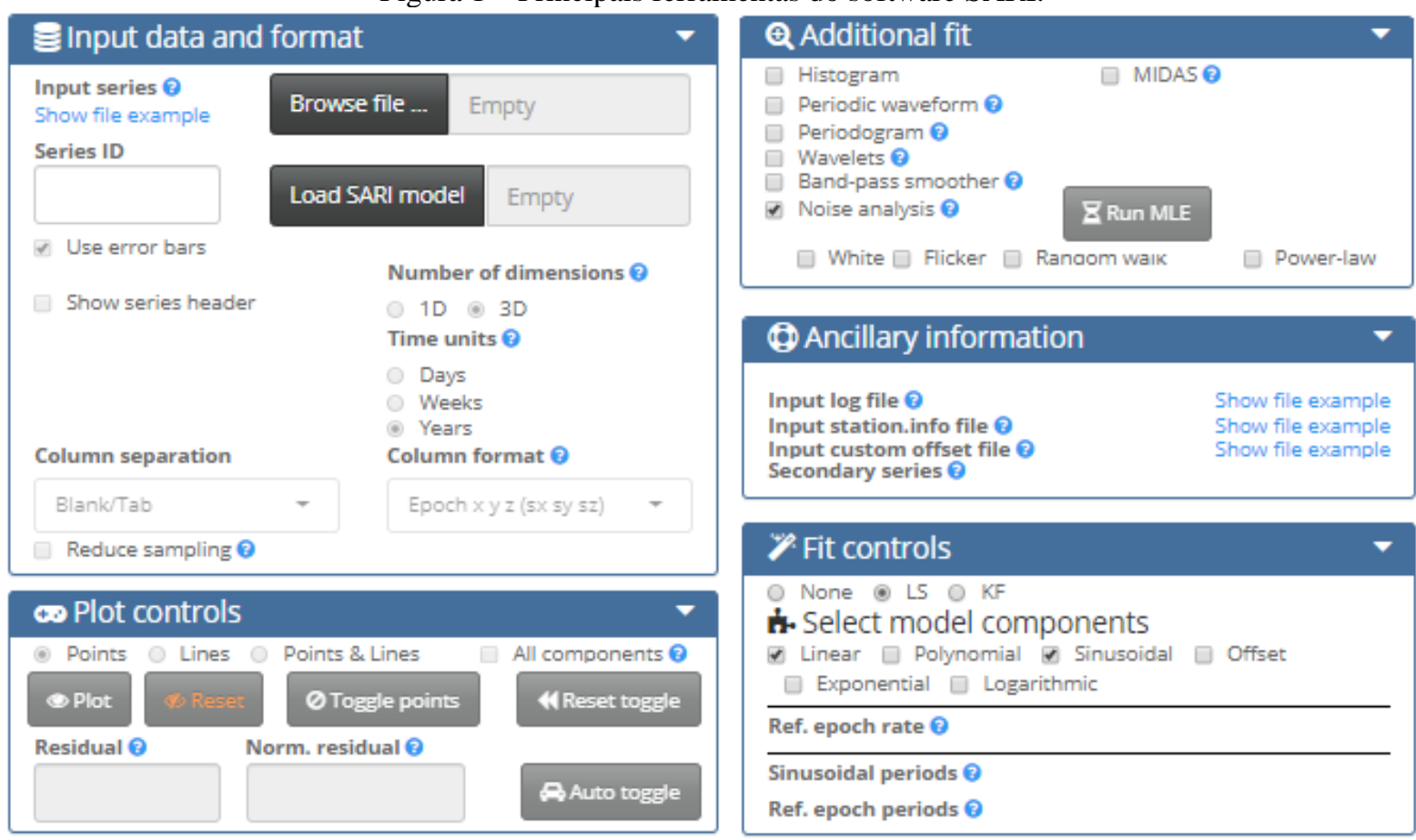

Fonte: Adaptada de Santamaría-Gómez (2019).

A aba de ajustes adicionais do SARI possui algumas ferramentas importantes, a saber: (SANTAMARÍA-GÓMEZ, 2019):

a) MIDAS (Median Interannual Difference Adjusted for Skewness): é um estimador de tendências automatizado, tendo sido implementado com base na descrição de Blewitt et al. (2016);

b) Histograma dos resíduos: realiza avaliação de estacionariedade usando os testes ADF (Augmented Dickey-Fuller) e KPSS (Kwiatkowski-Phillips-Schmidt-Shin);

c) Periodic waveform: É estimada a partir da série residual assumindo a estacionariedade da série temporal;

d) Periodograma: utiliza o periodograma de Lomb-Scargle e, no caso de amostragem irregular da série, o período de Nyquist é definido por Eyer e Bartholdi (1999).

e) Wavelet: calcula o produto interno da série e a wavelet de Morlet com valor complexo na qual é dimensionada e representada com frequência central igual a $2 \pi$ radianos, conforme descrito em Keitt (2008);

f) band-pass smoother: Foi implementado com base na descrição de Vondrák (1977). Aceita dois períodos de entrada: o passa-baixo e o passa-alta. Esta ferramenta reduz a variabilidade em torno dos períodos escolhidos, a partir de observações amostradas de forma irregular, não preenchidas, não interpoladas e incertas ou de amostras uniformes com intervalos de erros variáveis;

g) Análise de ruídos (noise analysis): Foi implementado conforme Williams (2003) e Bos et al. (2013). As análises de ruídos são estimadas por meio da MLE e possuem quatro processos estocásticos: ruído branco, ruído de cintilação, ruído de passeio aleatório e ruído da lei de potência. A lei de potência é uma generalização dos outros três processos e, portanto, não é possível estimar a lei de potência com oscilação ou passeio aleatório, mas é compatível com o ruído branco.

O SARI possui uma vasta possibilidade de processamento de séries temporais de forma interativa. Salienta-se que o tempo de processamento aumenta consideravelmente para séries longas, com alta amostragem e modelos de ruído complexos. Séries longas podem ser ajustadas, mas o benefício interativo é perdido. Portanto, para economizar tempo de processamento, é altamente recomendável reduzir a amostragem da série ao mínimo necessário (SANTAMARÍA-GÓMEZ, 2019). Santamaría-Gómez (2019) recomenda amostragem semanal nas análises de séries temporais longas de coordenadas obtidas com GNSS. 


\section{MATERIAIS E MÉTODOS}

\subsection{Materiais}

A pesquisa foi realizada com os dados da RBMC coletados por receptores localizados na porção da placa tectônica Sul-Americana, localizada em território brasileiro. Foram utilizados dados diários de observáveis GNSS, no formato RINEX (Receiver Independent Exchange Format), de 94 estações, no período de 01/01/2012 a 29/12/2018, como mostra a Tabela 1.

Tabela 1 - Intervalo de tempo para aquisição dos dados.

\begin{tabular}{l|c|c|c|c|c|c}
\hline & Datas & Semana GPS & Época & Época média & Total de dias & Total de Semanas \\
\hline Inicio & $01 / 01 / 2012$ & 1669 & $2012,00(000 / 365)$ & 2015,50 & 2554 & 365 \\
Fim & $29 / 12 / 2018$ & 2033 & $2018,99(363 / 365)$ & & & \\
\hline
\end{tabular}

Fonte: Os autores (2021).

É importante destacar que os resultados obtidos no IBGE-PPP, de acordo com o período de dados processados (Tabela 1) estão referenciados à três materializações distintas: IGS08 (International GNSS Service - 17/04/2011 a 06/10/2012), IGb08 (07/10/2012 a 28/01/2017) e IGS14 (29/01/2017 a 16/05/2020) (IBGE, 2017).

O critério utilizado na escolha das estações foi de acordo com a disponibilidade de dados. Foram escolhidas todas as estações com, pelo menos, quatro anos de dados para o período supracitado. Ressalta-se que a distribuição geográfica das estações não são homogenias, sendo possível perceber algumas regiões com poucas estações e outras com alta densidade. As estações utilizadas nesta pesquisa são apresentadas na Figura 2, onde também são apresentadas as porcentagens de dados RINEX das estações utilizadas.

Figura 2 - Porcentagem de dados RINEX das estações selecionadas no estudo, no período de 01/01/2012 a 29/12/2018.

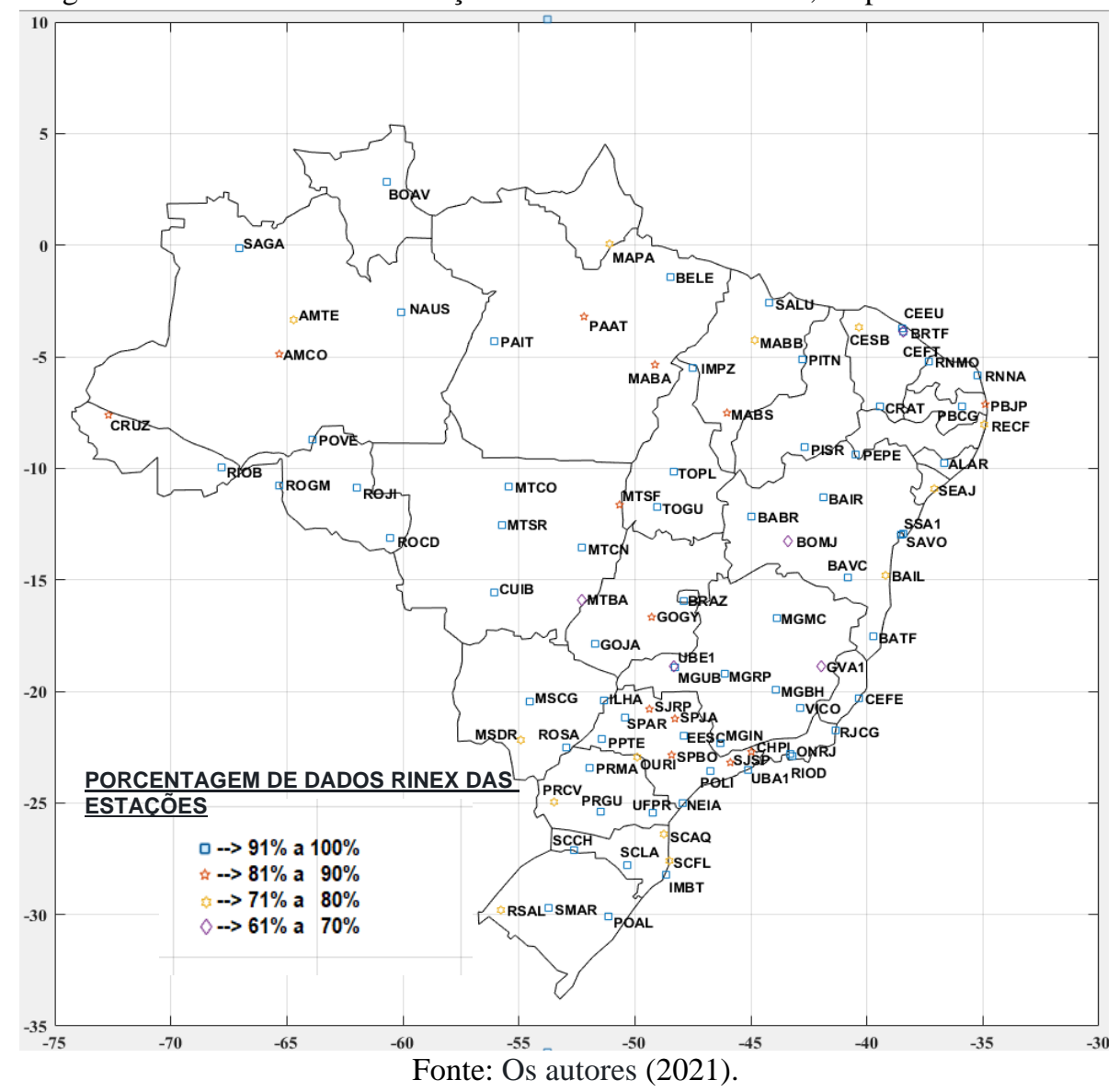

Além do software SARI e do serviço online IBGE-PPP, também foram utilizados o Matlab (versão 
2012b) e o RINEX EDITION (NASCIMENTO et al., 2017).

\subsection{Métodos}

Os principais passos metodológicos empregados nesta pesquisa são representados pela Figura 3. Os arquivos RINEX (24 h) foram pós-processados pelo método de PPP, modo estático, pelo serviço on-line IBGEPPP com suporte do software RINEX EDITION (NASCIMENTO et al., 2017), que auxiliou na realização dos downloads e processamentos dos arquivos RINEX, bem como na realização das extrações das informações dos arquivos de processamentos, de forma semiautomatizada.

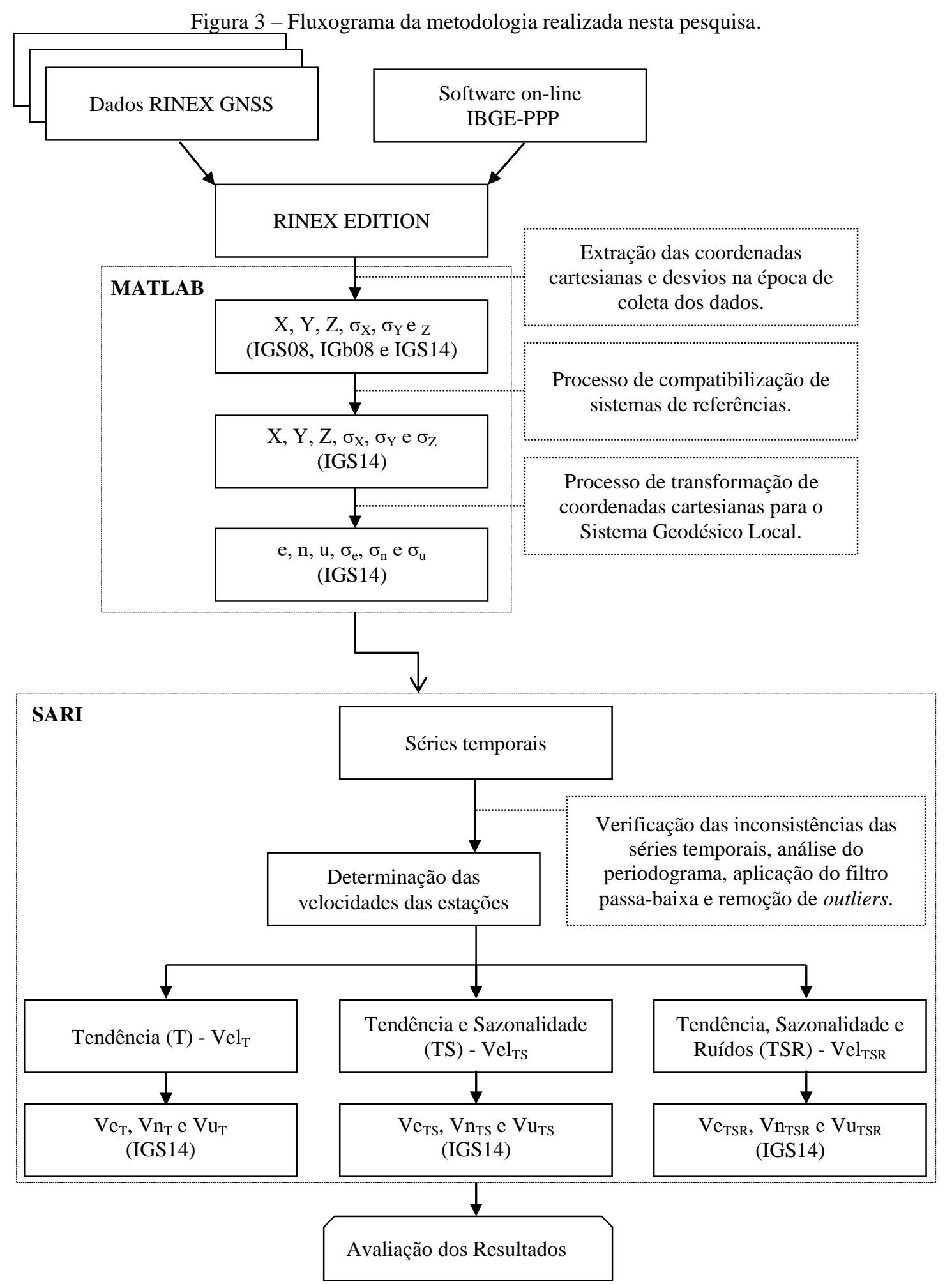

Fonte: Os autores (2021). 
Depois do pós-processamento dos dados GNSS, foram realizadas as compatibilizações dos referenciais das coordenadas para a atual materialização do IGS (IGS14). É importante frisar que, ao término do processamento, foram obtidas as coordenadas cartesianas geocêntricas e respectivas precisões $\left(\mathrm{X}, \mathrm{Y}, \mathrm{Z}, \sigma_{\mathrm{X}}\right.$, $\left.\sigma_{\mathrm{Y}}, \sigma_{\mathrm{Z}}\right)$ para todas as estações analisadas. Essas coordenadas e precisões foram transformadas para o SGL (Sistema Geodésico Local - e, n, u, $\sigma_{e}, \sigma_{n}, \sigma_{u}$ ). Estes processamentos foram realizados a partir de rotinas implementadas no software Matlab (versão 2012b).

As coordenadas diárias estimadas para cada componente durante um período representam as séries temporais das estações, ou seja, cada estação gerou três séries temporais - uma série para cada componente do SGL.

Após a etapa de determinação das coordenadas no SGL, foram realizados três experimentos para determinação das velocidades das estações, nos quais as séries temporais foram ajustadas com uso do software SARI pelo MMQ. As três soluções realizadas com o programa SARI são: 1) considerando apenas a tendência da série temporal ( $\left.\mathrm{Vel}_{\mathrm{T}}\right)$; 2) considerando a 1) mais efeitos sazonais ( $\mathrm{Vel}_{\mathrm{TS}}$ ); e 3) considerando a 2) mais efeitos de ruídos $\left(\mathrm{Vel}_{\mathrm{TSR}}\right)$. Por fim, foram salvos os arquivos de cada um dos três processamentos para posterior análise dos resultados.

Segundo Santamaria-Gómez (2019), não apenas a tendência e sazonalidade precisam ser analisadas em uma série de coordenadas GNSS, mas também as propriedades do ruído, sendo o método de estimativa de máxima verossimilhança (MLE) amplamente utilizado para esse tipo de análise. Sendo assim, os índices espectrais (k) dos ruídos da lei de potência foram calculados usando-se a estimativa de máxima verossimilhança no experimento Vel $\mathrm{TSR}_{\mathrm{T}}$.

Ao realizar o processamento das séries de coordenadas obtidas com GNSS, inicialmente foram carregados os dados das séries e selecionado o tipo de ajuste relativo a cada experimento. Em seguida foi realizada a remoção de outliers. Salienta-se que a detecção de outlier é uma parte importante, uma vez que eles podem interferir na obtenção das velocidades de estações GNSS e dificultar as análises de outros sinais nas séries temporais (GEIRSSON, 2003). Os outliers podem ser causados, dentre outros fatores, pela presença de arquivos de dados com falhas.

Nesta pesquisa, os outliers foram removidos depois de plotar o periodograma dos resíduos e realizar a aplicação do filtro passa-baixa de Vondrák (1977), conforme recomendado por Santamaría-Gómez (2019). Após a combinação do uso do periodograma e do filtro passa-baixa, foi realizado, de forma interativa, a remoção dos outliers de acordo com as características de cada série temporal. Ressalta-se que o SARI realiza, de forma automática, o novo processamento posteriormente à exclusão dos outliers.

Para Klos, Bos e Bogusz (2018), os ruídos têm influência significativa na incerteza das velocidades. Os autores ainda afirmam que qualquer sinal sazonal ou periodicidade residual não modelado e removido adequadamente, irá mover a parte estocástica para um ruído muito mais correlacionado, fazendo com que as incertezas sejam superestimadas. Além disso, a solução $\mathrm{Vel}_{\mathrm{TSR}}$ apresentou as menores discrepâncias dimensionais médias, quando comparado com a solução multianual do SIRGAS-CON realização SIR17P01 (época 2015). Dessa forma, assumindo-se que as velocidades obtidas pelo experimento Vel ${ }_{\mathrm{TSR}}$ correspondem aos valores mais confiáveis para as estações, este experimento foi comparado com os demais. A realização SIR17P01 está associada à época de referência de 17/04/2011 até 28/01/2017, alinhado também ao IGS14. Maiores informações podem ser obtidas em (SÁNCHEZ; DREWES, 2020) ou no site do SIRGAS (http://www.sirgas.org/pt/sirgas-con-network/coordinates/multi-year-solutions/).

\section{RESULTADOS E DISCUSSÃO}

Inicialmente, serão apresentadas as velocidades das 94 estações analisadas nesta pesquisa e obtidas a partir dos três experimentos realizados $\left(\mathrm{Vel}_{\mathrm{T}}, \mathrm{Vel}_{\mathrm{TS}}\right.$ e $\left.\mathrm{Vel}_{\mathrm{TSR}}\right)$, conforme apresenta a Tabela 2. 
Tabela 2 - Velocidades das 94 estações da RBMC analisadas nesta pesquisa a partir dos três experimentos $\left(\operatorname{Vel}_{\mathrm{T}}\right.$, $\mathrm{Vel}_{\mathrm{TS}}$ e $\left.\mathrm{Vel}_{\mathrm{TSR}}\right)$.

\begin{tabular}{|c|c|c|c|c|c|c|c|c|c|}
\hline & \multicolumn{3}{|c|}{$\mathbf{V e l}_{\mathbf{T}}-(\mathbf{m m} / \mathbf{a n o})$} & \multicolumn{3}{|c|}{$\mathbf{V e l}_{\mathrm{TS}}-(\mathrm{mm} / \mathbf{a n o})$} & \multicolumn{3}{|c|}{$\mathrm{Vel}_{\text {TSR }}-(\mathrm{mm} / \mathrm{ano})$} \\
\hline & $\mathbf{V e}_{\mathbf{T}}$ & \begin{tabular}{|l|}
$\mathbf{V n}_{\mathbf{T}}$ \\
\end{tabular} & $\mathbf{V} \mathbf{u}_{T}$ & $\mathrm{Ve}_{\mathrm{TS}}$ & \begin{tabular}{|l|}
$\mathbf{V n}$ \\
TS
\end{tabular} & $\mathbf{V u} \mathbf{u}_{\mathrm{TS}}$ & $V_{e_{T S R}}$ & \begin{tabular}{|l|}
$\mathbf{V n}$ \\
TSR
\end{tabular} & $\mathbf{V u} \mathbf{u}_{\mathrm{TSR}}$ \\
\hline & $-4,07 \pm 0,04$ & $12,71 \pm 0,02$ & $0,20 \pm 0,06$ & $|-4,09 \pm 0,04|$ & $12,69 \pm 0,02$ & $\mathbf{0 , 1 8} \pm \mathbf{0 , 0 6}$ & $-4,13 \pm 0,25 \mid$ & $12,66 \pm 0,34$ & $\mathbf{0 , 1 4} \pm \mathbf{0 , 3 8}$ \\
\hline МCO & $-3,18 \pm 0,07$ &, $80 \pm 0$ & $23 \pm 0$ & $, 14 \pm 0,06$ & $1,77 \pm 0$ & $\mathbf{0 , 0 1} \pm \mathbf{0 , 0 9}$ & $-3,18 \pm 0,23$ & $11,78 \pm 0,56$ & $-0,02 \pm$ \\
\hline AMTE & $-5,91 \pm 0,09$ & $11,56 \pm 0,08$ & $2,45 \pm 0,31$ & $\mathbf{7 , 9 9} \pm \mathbf{0 , 0 8}$ & $11,53 \pm 0,07$ & $2,76 \pm 0,16$ & $-6,00 \pm 0,29 \mid$ & $11,50 \pm 0,67$ & $2,71 \pm 1$ \\
\hline BAB B R & $-3,11 \pm 0,04$ & $12,96 \pm 0,04$ & $3,65 \pm 0,09$ & $|-3,16 \pm 0,04|$ & $13,02 \pm 0,04$ & $\mathbf{3 , 3 7} \pm \mathbf{0 , 0 8}$ & $-3,18 \pm 0,25 \mid$ & $13,02 \pm 0,48$ & $3,38 \pm($ \\
\hline B AIL & $-3,17 \pm 0,08$ & $3,71 \pm 0,06$ & $-\mathbf{0 , 0 7} \pm \mathbf{0 , 1 3}$ & $\mathbf{3 , 1 7} \pm \mathbf{0 , 0 8}$ & $13,70 \pm 0,06$ & $-0,11 \pm 0,13 \mid$ & $-3,24 \pm 0,46$ & $13,67 \pm 0,67$ & $-\mathbf{0 , 1 1} \pm \mathbf{1}$ \\
\hline BAIR & $-5,04 \pm 0,05$ & $2,29 \pm 0,04$ & $\mathbf{1 , 5 3} \pm \mathbf{0 , 0 7}$ & $|-5,07 \pm 0,05|$ & $12,29 \pm 0,04$ & $1, \mathbf{4 3} \pm \mathbf{0 , 0 7}$ & $-5,10 \pm 0,36$ & $12,28 \pm 0,45$ & $1,41 \pm 0,62$ \\
\hline BATF & $-2,65 \pm 0,07$ & $3,11 \pm 0,04$ & $-1,97 \pm 0,20 \mid$ & $-2,65 \pm 0,06 \mid$ & $13,12 \pm 0,04$ & $|-1,99 \pm 0,19|$ & $-2,70 \pm 0,49$ & $13,08 \pm 0,50$ & $-1,67 \pm 1,51$ \\
\hline BAVC & $-4,50 \pm 0,03$ & $13,64 \pm 0,02$ & $\mathbf{0 , 5 4} \pm \mathbf{0 , 0 6}$ & $-4,57 \pm 0,03$ & $13,64 \pm 0,02$ & $\mathbf{0 , 4 3} \pm \mathbf{0 , 0 6}$ & $-4,72 \pm 0,31 \mid$ & $13,72 \pm 0,57$ & $\mathbf{0 , 4 0} \pm \mathbf{0 , 7 9}$ \\
\hline BELE & $-4,36 \pm 0,04$ & $13,36 \pm 0,03$ & $1,95 \pm 0,10$ & $-4,41 \pm 0,04 \mid$ & $13,29 \pm 0,03$ & $1,64 \pm 0,08$ & $-4,43 \pm 0,22$ & $13,26 \pm 0,35$ & $1,63 \pm 0,50$ \\
\hline BOAV & $-4,06 \pm 0,03$ & $2,20 \pm 0,04$ & $1,19 \pm 0,09$ & $-4,04 \pm 0,03$ & $12,18 \pm 0,03$ & $1,18 \pm 0,06$ & $-4,08 \pm 0,19 \mid$ & $12,20 \pm 0,31$ & $1,20 \pm 0,75$ \\
\hline ВОМJ & $-4,71 \pm 0,07$ & $2,93 \pm 0,06$ & $3,33 \pm 0,14$ & $-4,80 \pm 0,06$ & $12,95 \pm 0,06$ & $3,04 \pm 0,11$ & $-4,82 \pm 0,36$ & $12,96 \pm 0,74$ & $3,04 \pm \mathbf{0 , 6 5}$ \\
\hline BRAZ & $-1,96 \pm 0,05$ & $12,34 \pm 0,03$ & $-1,01 \pm 0,12$ & $2,01 \pm 0,05$ & $12,32 \pm 0,03$ & $-1,01 \pm 0,10 \mid$ & $-2,05 \pm 0,60$ & $12,28 \pm 0,54$ & $-1,03 \pm 1$ \\
\hline BRTF & $-4,46$ & $4,54 \pm 0,04$ & $\mathbf{1 , 8 3} \pm \mathbf{0 , 0 8}$ & $4,60 \pm 0,05$ & $14,47 \pm 0,04$ & $1,37 \pm 0,08$ & $-4,61 \pm 0,29$ & $14,48 \pm 0,78$ &, $42 \pm$ \\
\hline CEEU & $-5,76 \pm 0,05$ & $2,20 \pm 0,04$ & $\mathbf{0 , 2 7} \pm \mathbf{0 , 0 6}$ & $\mathbf{5 , 8 0} \pm \mathbf{0 , 0 5}$ & $12,20 \pm 0,04$ & $\mathbf{0 , 2 0} \pm \mathbf{0 , 0 5}$ & $-5,84 \pm 0,28$ & $12,21 \pm 0,38$ & $\mathbf{0 , 1 8} \pm$ \\
\hline CEFE & $-3,84 \pm 0,05$ & $13,06 \pm 0,04$ & $1,35 \pm 0,07$ & $-\mathbf{3 , 8 6} \pm \mathbf{0 , 0 5}$ & $13,08 \pm 0,04$ & $1,30 \pm 0,07$ & $-3,89 \pm 0,26$ & $13,08 \pm 0,46$ & $1,28 \pm 0$ \\
\hline CEFT & $-5,76 \pm 0,07$ & $12,62 \pm 0,05$ & $-1,41 \pm 0,08 \mid$ & $|-5,77 \pm \mathbf{0 , 0 7}|$ & $12,65 \pm 0,05$ & $-1,42 \pm 0,08$ & $-5,84 \pm 0,49$ & $12,56 \pm 0,77$ & $-1,55 \pm 0,58$ \\
\hline CESB & $-4,60 \pm 0,07$ & $13,28 \pm 0,06$ & $-3,19 \pm 0,12$ & $-4,64 \pm 0,07 \mid$ & $13,30 \pm 0,06$ & $|-3,34 \pm 0,10|$ & $-4,68 \pm 0,36$ & $13,28 \pm 0,66$ & $-3,37 \pm 0,68$ \\
\hline CHPI & $-3,62 \pm 0,05$ & $12,65 \pm 0,03$ & $\mathbf{1 , 2 4} \pm \mathbf{0 , 0 9}$ & $|-3,64 \pm 0,05|$ & $12,69 \pm 0,03$ & $1,09 \pm 0,08$ & $-\mathbf{3 , 7 0} \pm \mathbf{0 , 3 1}$ & $12,69 \pm 0,50$ & $1,06 \pm 0,44$ \\
\hline CRAT & $-4,98 \pm 0,07$ & $13,28 \pm 0,05$ & $-\mathbf{0 , 2 5} \pm \mathbf{0 , 1 1} \mid$ & $|-5,01 \pm 0,07|$ & $13,26 \pm 0,05$ & $-\mathbf{0 , 3 3} \pm \mathbf{0 , 1 0} \mid$ & $|-5,04 \pm 0,44|$ & $13,26 \pm 0,37$ & $-\mathbf{0 , 3 7} \pm \mathbf{0 , 9 8}$ \\
\hline CRUZ & $-2,91 \pm \mathbf{0 , 0 6}$ & $10,96 \pm 0,05$ & $\mathbf{- 1 , 9 8} \pm \mathbf{0 , 1 2}$ & $|-2,90 \pm \mathbf{0 , 0 5}|$ & $10,94 \pm 0,05$ & $|-1,99 \pm 0,09|$ & $|-2,92 \pm 0,37|$ & $10,92 \pm 0,48$ & $-2,06 \pm 0$ \\
\hline CUIB & $-0,91 \pm 0,06$ & $11,00 \pm 0,06$ & $0,16 \pm 0,12$ & $-0,92 \pm 0,06$ & $11,05 \pm 0,05$ & $-0,14 \pm 0,09 \mid$ & $-0,96 \pm 0,55$ & $11,02 \pm 1,09$ & $-0,18 \pm 0$ \\
\hline EESC & $-4,19 \pm 0,04$ & $12,35 \pm 0,03$ & $0,73 \pm 0,08$ & $-4,20 \pm 0,04$ & $12,35 \pm 0,03$ & $\mathbf{0 , 5 2} \pm \mathbf{0 , 0 7}$ & $-4,22 \pm 0,30$ & $12,33 \pm 0,52$ & $\mathbf{0 , 4 9} \pm \mathbf{0 , 7 8}$ \\
\hline GOGY & $-3,71 \pm 0,05$ & $12,32 \pm 0,06$ & $3,89 \pm 0,14$ & $|-3,72 \pm 0,05|$ & $12,36 \pm 0,05$ & $3,62 \pm 0,11$ & $-3,74 \pm 0,38$ & $12,34 \pm 0,49$ & $3,62 \pm$ \\
\hline GOJA & $-3,14 \pm 0,05$ & $11,83 \pm \mathbf{0 , 0 4}$ & $-1,27 \pm 0$ & $-\mathbf{3 , 1 4} \pm \mathbf{0 , 0 5}$ & $11,84 \pm \mathbf{0 , 0 4}$ & $-1,41 \pm 0,07 \mid$ & $-\mathbf{3 , 1 7} \pm \mathbf{0 , 3 0}$ & $11,82 \pm 0,47$ & $-1,42 \pm$ \\
\hline GVA1 & $-3,69 \pm 0,08$ & $13,00 \pm 0,05$ & $\mathbf{0 , 2 0} \pm \mathbf{0}$ & $-3,78 \pm 0,08$ & $13,00 \pm 0,05$ & $-\mathbf{0 , 0 9} \pm \mathbf{0 , 1 3} \mid$ & $-3,83 \pm 0,64 \mid$ & $12,98 \pm 0,92$ & $-0,07 \pm$ \\
\hline ILHA & $-2,65 \pm 0,03$ & $12,47 \pm 0,04$ & $\mathbf{0 , 1 0} \pm \mathbf{0}$ & $-2,66 \pm 0,03 \mid$ & $12,45 \pm 0,04$ & $\mathbf{0 , 3 0} \pm \mathbf{0 , 1 0}$ & $-2,71 \pm 0,30 \mid$ & $12,44 \pm 0,47$ & $\mathbf{0 , 2 8} \pm$ \\
\hline IMB T & $-3,63 \pm 0,05$ & $12,49 \pm 0,03$ & $\mathbf{- 0 , 5 4} \pm \mathbf{0 , 0 6}$ & $-3,65 \pm 0,05 \mid$ & $12,51 \pm 0,03$ & $-0,52 \pm 0,06 \mid$ & $-3,79 \pm 0,45 \mid$ & $12,47 \pm 0,60$ & $-0,61 \pm$ \\
\hline IMPZ & $-4,41 \pm 0,04$ & $12,59 \pm 0,03$ & $-1,17 \pm 0,08$ & $-4,43 \pm 0,04 \mid$ & $12,59 \pm 0,03$ & $-1,33 \pm 0,06$ & $-4,44 \pm 0,19 \mid$ & $12,57 \pm 0,28$ & $-1,34 \pm$ \\
\hline МАВА & $-3,03 \pm 0,05$ & $12,37 \pm 0,03$ & $-1,17 \pm 0,11$ & $-3,04 \pm 0,05 \mid$ & $12,37 \pm 0,03$ & $-1,29 \pm 0,07 \mid$ & $-3,09 \pm 0,31 \mid$ & $12,36 \pm 0,34$ & $-1,30 \pm$ \\
\hline МАВ В & $-2,76 \pm 0,07$ & $13,01 \pm 0,05$ & $1,67 \pm 0,15$ & $85 \pm 0,07$ & $12,97 \pm 0,05$ & $1,06 \pm 0,12$ & $-2,88 \pm 0,38$ & $12,97 \pm 0,51$ & $1,07 \pm$ \\
\hline MABS & $-5,28 \pm 0,07$ & $12,22 \pm 0,04$ & $2,56 \pm 0,16$ & $-\mathbf{- 5 , 3 3} \pm \mathbf{0 , 0 7}$ & $12,21 \pm 0,04$ & $2,30 \pm 0,13$ & $-5,39 \pm 0,30 \mid$ & $12,21 \pm 0,38$ & $2,29 \pm 1$ \\
\hline MAPA & $-3,90 \pm 0,06$ & $13,01 \pm 0,04$ & $-0,01 \pm 0,12 \mid$ & $, 92 \pm 0,06$ & $12,98 \pm 0,04$ & $-0,27 \pm 0,09 \mid$ & $-3,94 \pm 0,32$ & $12,94 \pm 0,48$ & $-0,32=$ \\
\hline MGBH & $-5,26 \pm 0,04$ & $12,19 \pm 0,03$ & $2,93 \pm 0,10$ & $-5,28 \pm 0,04$ & $12,20 \pm 0,03$ & $2,83 \pm \mathbf{0 , 0 9}$ & $-5,43 \pm 0,46 \mid$ & $12,17 \pm 0,58$ & $2,94 \pm 1$ \\
\hline MGIN & $-3,49 \pm 0,03$ & $13,06 \pm 0,03$ & $1,05 \pm 0$ & $-3,51 \pm 0,03$ & $13,08 \pm 0,03$ & $\mathbf{0 , 9 5} \pm \mathbf{0 , 0 6}$ & $-3,53 \pm 0,26$ & $13,08 \pm 0,42$ & 0,94 \\
\hline MGMC & $-4,03 \pm 0,03$ & $13,79 \pm 0,04$ & $5,34 \pm$ & $, 06 \pm 0,03$ & $13,81 \pm 0,04$ & $5,25 \pm 0,06$ & $-4,08 \pm 0,25$ & $13,80 \pm 0,48$ & 5,23 \\
\hline MGRP & $-3,07 \pm 0,04$ & $12,44 \pm 0,03$ & $2,88 \pm 0,10$ & $, 09 \pm 0,04$ & $12,45 \pm 0,03$ & $2,70 \pm 0,09$ & $-3,14 \pm 0,24$ & $12,43 \pm 0,51$ & 2,69 \\
\hline MGUB & $-3,53 \pm 0,03$ & $12,54 \pm 0,03$ & $2,36 \pm 0,08$ & $, 56 \pm 0,03$ & $12,58 \pm 0,03$ & $\mathbf{2 , 0 5} \pm \mathbf{0 , 0 7}$ & $-3,56 \pm 0,27$ & $12,59 \pm 0,40$ & $2,01 \pm 0,60$ \\
\hline MSCG & $-2,32 \pm 0,04$ & $12,55 \pm 0,06$ & $-3,43 \pm 0,12$ & $-2,29 \pm 0,04 \mid$ & $12,67 \pm 0,06$ & $-3,57 \pm 0,11 \mid$ & $-2,33 \pm 0,33$ & $12,65 \pm 0,62$ & $-3,62 \pm 1,16$ \\
\hline MSDR & $-3,10 \pm 0,05$ & $11,40 \pm 0,05$ & $-2,09 \pm 0,12$ & $-3,10 \pm 0,05 \mid$ & $11,40 \pm 0,05$ & $-2,07 \pm 0,10 \mid$ & $-3,13 \pm 0,35$ & $11,36 \pm 0,54$ & $-2,10$ \\
\hline MTBA & $-2,99 \pm 0,05$ & $12,75 \pm 0,05$ & $-0,09 \pm 0,16 \mid$ & $2,99 \pm 0,05$ & $12,89 \pm 0,04$ & $-0,44 \pm 0,11 \mid$ & $-3,03 \pm 0,36$ & $12,87 \pm 0,55$ & $-0,45 \pm 0,65$ \\
\hline MTCN & $-3,63 \pm 0,04$ & $13,60 \pm 0,04$ & $\mathbf{1 , 5 6} \pm \mathbf{0 , 1 0}$ & $-3,64 \pm 0,04$ & $13,66 \pm 0,04$ & $\mathbf{1 , 1 6} \pm \mathbf{0 , 0 7}$ & $-3,68 \pm 0,22$ & $13,68 \pm 0,50$ & $1,16 \pm 0,50$ \\
\hline MTCO & $-3,50 \pm 0,04$ & $12,90 \pm 0,04$ & $3,02 \pm$ & $-3,50 \pm 0,04$ & $12,92 \pm 0,04$ & $2,79 \pm 0,06$ & $-3,65 \pm 0,18$ & $12,93 \pm 0,47$ & $2,90 \pm 0,69$ \\
\hline MTSF & $-3,74 \pm 0,05$ & $12,76 \pm 0,03$ & $1,21=$ & $|-3,74 \pm 0,05|$ & $12,82 \pm 0,03$ & $1,20 \pm 0,06$ & $|-3,76 \pm 0,24|$ & $12,81 \pm \mathbf{0 , 4 0}$ & $\pm 0,60$ \\
\hline MTSR & $-4,20 \pm 0,05$ & $13,00 \pm 0,04$ & $\mathbf{0 , 5 3} \pm \mathbf{0 , 1 3}$ & $|-4,21 \pm 0,05|$ & $13,01 \pm \mathbf{0 , 0 3}$ & $0,21 \pm 0,09$ & $-4,25 \pm 0,27 \mid$ & $13,01 \pm 0,34$ & $0,20 \pm 0,71$ \\
\hline NAUS & $-4,21 \pm 0,07$ & $\mathbf{3 , 5 5} \pm \mathbf{0 , 0 8}$ & $1,34 \pm 0,24$ & $, 19 \pm 0,07$ & $13,42 \pm 0,07$ & $\mathbf{0 , 7 7} \pm \mathbf{0 , 1 3}$ & $-4,25 \pm 0,34 \mid$ & $13,43 \pm 1,35$ & $\pm \mathbf{1 , 5 6}$ \\
\hline NEIA & $-\mathbf{1 , 5 8} \pm \mathbf{0 , 0 5}$ & $1,90 \pm$ & $1,39 \pm 0,19$ & $|-1,58 \pm 0,05|$ & $11,92 \pm \mathbf{0 , 0 4}$ & $1,39 \pm 0$ & $-\mathbf{- 1 , 5 8} \pm \mathbf{0 , 3 8}$ & $11,89 \pm 0,54$ & 92 \\
\hline ONRJ & $-2,88 \pm \mathbf{0 , 0 5}$ & $\mathbf{0 , 0 4}$ & 4,11 & $, 91 \pm 0,05$ & $13,38 \pm 0,04$ & $4,00 \pm 0$ & $-3,11 \pm 0,42 \mid$ & $13,40=$ & $\mathbf{3 , 8 8}$ \\
\hline OURI & $-2,13 \pm 0$ & 1 & $-3,85$ & $17 \pm 0,09$ & $12,01 \pm 0$ & $-3,85 \pm 0$ & $-2,18 \pm 0,45$ & 12, & $-3,93$ \\
\hline & & & &, 10 & & & $2 \pm 0,27$ & & \\
\hline & & & & ,09 & & & $5 \pm 0,30$ & & \\
\hline & & & & ,08 & & & $3 \pm 0,19$ & & \\
\hline & & & & $2 \pm 0,13$ & $14,46 \pm 0,07$ & & $62 \pm 0,42$ & & \\
\hline & $-4,71 \pm 0,05$ & & & $-4,78 \pm 0,18$ & $12,84 \pm 0,04$ & 0,67 & $-4,86 \pm 0,33$ & 12,86 & \\
\hline & $-4,65 \pm 0,06$ & $13,00 \pm 0$ & 1,1 & $-4,69 \pm 0,08 \mid$ & $13,00 \pm 0,03$ & 1,10 & $-4,69 \pm 0,30 \mid$ & 13,01 & $\mathbf{0 , 9 7}$ \\
\hline & $-3,89 \pm 0,04$ & $12,68 \pm 0$ & $1,02 \pm$ & $-3,92 \pm 0,07$ & $12,66 \pm 0,03$ & $\mathbf{1 , 0 2} \pm \mathbf{0 , 0 5}$ & $-3,93 \pm 0,22$ & $12,66=$ & $\mathbf{0 , 8 6} \pm$ \\
\hline POAL & $-2,97 \pm 0,05$ & $11,85 \pm 0,04$ & $-0,71 \pm 0,08$ & $-2,99 \pm 0,12$ & $11,91 \pm 0,03$ & $-0,71 \pm \mathbf{0 , 0 6}$ & $-3,06 \pm 0,38$ & $11,91 \pm 0,53$ & $-0,79 \pm 0,76$ \\
\hline & $-1,31 \pm 0,05$ & $13,74 \pm 0,07$ & $-1,78 \pm 0,11$ & $-1,33 \pm 0,08$ & $13,79 \pm 0,07$ & $-1,77 \pm 0,09$ & $-1,35 \pm 0,57$ & $13,77 \pm 0,68$ & $-2,01 \pm 1,00$ \\
\hline POVE & $-3,01 \pm 0,04$ & $|12,54 \pm 0,04|$ & $\mathbf{1 , 0 5} \pm \mathbf{0 , 1 2}$ & $|-2,97 \pm \mathbf{0 , 1 6}|$ & $12,63 \pm 0,06$ & $\mathbf{0 , 9 2} \pm \mathbf{0 , 0 6}$ & $-2,88 \pm 0,23$ & $12,59 \pm 0,42$ & $\mathbf{0 , 8 1} \pm \mathbf{0 , 6 5}$ \\
\hline & $-3,05 \pm 0,03$ & $12,27 \pm \mathbf{0 , 0 3}$ & $|-1,07 \pm \mathbf{0 , 0 8}|$ & $|-3,06 \pm 0,04|$ & $12,30 \pm 0,03$ & $-1,07 \pm 0,05 \mid$ & $|-3,07 \pm 0,23|$ & $12,31 \pm 0,39$ & $-1,18 \pm 0,75$ \\
\hline PRCV & $-3,33 \pm 0,05$ & $13,97 \pm 0,06$ & $0,56 \pm 0,10$ & $-3,38 \pm 0,10$ & $13,96 \pm 0,07$ & $\mathbf{0 , 6 7} \pm \mathbf{0 , 0 9}$ & $-3,38 \pm 0,49$ & $13,98 \pm 0,63$ & $\mathbf{0 , 6 1} \pm \mathbf{0 , 8 7}$ \\
\hline PRGU & $-4,51 \pm 0,04$ & $12,27 \pm 0,04$ & $-0,08 \pm 0,08$ & $-4,55 \pm 0,22$ & $12,46 \pm 0,07$ & $-\mathbf{- 0 , 2 0} \pm \mathbf{0 , 0 7}$ & $-4,71 \pm 0,48$ & $12,41 \pm \mathbf{0 , 8 3}$ & $-\mathbf{0 , 1 1} \pm \mathbf{0 , 8 3}$ \\
\hline PRMA & $-2,23 \pm 0,05$ & $13,39 \pm 0,03$ & $|-1,27 \pm 0,07|$ & $-2,25 \pm 0,05 \mid$ & $13,39 \pm 0,04$ & $-1,27 \pm \mathbf{0 , 0 7}$ & $-2,25 \pm 0,32 \mid$ & $13,41 \pm 0,43$ & $-1,42 \pm 0,69$ \\
\hline RECF & $-3,80 \pm \mathbf{0 , 0 6}$ & $14,91 \pm 0,06 \mid$ & $|-2,37 \pm \mathbf{0 , 1 0}|$ & $-\mathbf{- 3 , 8 6} \pm \mathbf{0 , 1 3}$ & $14,93 \pm 0,05$ & $-2,37 \pm \mathbf{0 , 0 8}$ & $|-3,88 \pm 0,46|$ & $14,93 \pm 0,65$ & $-2,38 \pm 0,62$ \\
\hline RIOB & $-1,93 \pm 0,07$ & $10,69 \pm 0,03$ & $\mathbf{0 , 2 8} \pm \mathbf{0 , 1 3}$ & $|-1,91 \pm 0,05|$ & $10,72 \pm 0,08$ & $\mathbf{0 , 3 1} \pm \mathbf{0 , 0 8}$ & $-\mathbf{- 1 , 9 3} \pm \mathbf{0 , 4 8}$ & $10,67 \pm 0,34$ & $\mathbf{0 , 0 5} \pm \mathbf{0 , 8 6}$ \\
\hline RIOD & $-4,57 \pm 0,04$ & $\mathbf{1 1 , 9 2} \pm \mathbf{0 , 0 5}$ & $1,52 \pm 0,11$ & $|-4,57 \pm 0,05|$ & $11,94 \pm 0,05$ & $1,52 \pm 0,09$ & $|-4,57 \pm 0,34|$ & $11,92 \pm 0,88$ & $1,47 \pm 1,03$ \\
\hline
\end{tabular}


(conclusão)

\begin{tabular}{|c|c|c|c|c|c|c|c|c|c|}
\hline & \multicolumn{3}{|c|}{$\mathrm{Vel}_{\mathrm{T}}-(\mathbf{m m} / \mathbf{a n o})$} & \multicolumn{3}{|c|}{$\mathrm{Vel}_{\mathrm{TS}}-(\mathrm{mm} / \mathrm{ano})$} & \multicolumn{3}{|c|}{$\mathbf{V e l}_{\mathrm{TSR}}-(\mathbf{m m} / \mathbf{a n o})$} \\
\hline & $\mathbf{V e}_{T}$ & $\mathbf{V} \mathbf{n}_{T}$ & $\mathbf{V} \mathbf{u}_{\mathrm{T}}$ & $\mathbf{V e}_{\mathrm{TS}}$ & $\mathbf{V n}_{\mathrm{TS}}$ & $\mathbf{V u} \mathbf{u}_{\mathrm{TS}}$ & $\mathrm{Ve}_{\mathrm{TSR}}$ & $\mathbf{V} n_{\text {TSR }}$ & $\mathbf{V u} \mathbf{u}_{\mathrm{TSR}}$ \\
\hline & $63 \pm 0$, & $, 10 \pm 0,03$ & 07 & $69 \pm 0,10$ & $2,09 \pm 0,1$ & $\overline{77 \pm 0,0}$ & $, 70 \pm 0,27$ & $12,10 \pm 0,50$ & \\
\hline & $56 \pm 0,08$ & $93 \pm 0,05$ & $-2,32 \pm 0,08$ & $, 65 \pm 0,17$ & $15,91 \pm 0,06$ & $-2,38 \pm 0,08$ & $-4,67 \pm 0,40$ & $15,89 \pm 0,41$ & \\
\hline NA & 4 & & $-0,65 \pm 0,09$ & $15 \pm 0$ & $36 \pm$ &, $59 \pm 1$ & $2,95 \pm 0,32$ &, 53 & \\
\hline & & 03 & $06 \pm 0,09$ &, $18 \pm 0$ & $11,74 \pm$ & $\mathbf{0 , 0 2} \pm \mathbf{0 , 0 5}$ & $-3,28 \pm 0,28$ & $11,63 \pm 0,44$ & \\
\hline GM & & 04 & $-1,11 \pm 0,12$ & $, 59 \pm 0,03$ & $11,01=$ & $-1,13 \pm 0,07$ & $-1,59 \pm 0,26$ & 11,02 & \\
\hline & & $\delta 2 \pm 0$ & $3,36 \pm 0,10$ & ,67 $\pm 0,12$ &, $92 \pm$ & $3,19 \pm 0$ & $-2,77 \pm 0,23$ & $\mathbf{0 , 3 6}$ & \\
\hline $\mathbf{S A}$ & & & $-2,27 \pm 0,08$ & $-3,31 \pm 0$ & 1 & $-2,27 \pm$ & $\mathbf{0 , 3 2}$ & 11, & \\
\hline & & & 11 & 99 & 1 & $40 \pm$ & $=0,61$ & & \\
\hline & & & 10 & & & & 0,26 & & \\
\hline & & & & & & &, 59 & & \\
\hline & & & & & & & & & \\
\hline & & & $-0,72 \pm$ & 0,10 & 12,35 & $-0,64 \pm$ & $-3,09 \pm 0,50$ & 12,36 & \\
\hline & & & & & & & & & \\
\hline & & 06 & & 0,05 & 12,17 & & $-3,90 \pm 0,36$ & 12 , & \\
\hline & & & & & & & $-3,08 \pm$ & & \\
\hline &, 06 &, 05 & $-1,9$ & $-4,71 \pm$ & 1 & $98 \pm$ & $-4,70 \pm 0,37$ &, 59 & \\
\hline & & & & & & & & & \\
\hline & $\mathbf{0 , 0 7}$ & $, 61 \pm 0,05$ & $-2,87 \pm 0,12$ & $-4,02 \pm 0,10$ & 12,62 & $, 90 \pm 0,10$ & $-4,04 \pm 0,50$ & 12,62 & \\
\hline & & & & & & -1 & 28 & & \\
\hline & 0,04 & $, 03 \pm 0,03$ & $-1,91 \pm 0,07$ & $-3,79 \pm 0,14$ & $12,09 \pm 0,05$ & $-1,72 \pm 0,06$ & $-3,88 \pm 0,31$ & $12,06 \pm 0,45$ & $-2,29$ \\
\hline & & & & & & & $39 \pm 0,39$ & & \\
\hline & & & $-0,81 \pm 0,10$ & $-3,70 \pm 0,10$ & & $-0,83 \pm 0$ & $-3,73 \pm 0,41$ & & \\
\hline & & & & -6 & & & & & \\
\hline & & & & & & & & & \\
\hline & & & & $3,93 \pm 0,36$ & & & $-3,57 \pm 0,21$ & & \\
\hline & & & & & & & & & \\
\hline & $4,45 \pm 0,18$ & $12,20 \pm 0,10$ & $-2,49 \pm 0,35$ & $-4,60 \pm 0,28$ & $12,28 \pm 0,11$ & $-2,44 \pm 0,26$ & $-4,58 \pm 0,66$ & $12,26 \pm 0,94$ & \\
\hline & & & & & $13,01 \pm 0,04$ & & $-3,66 \pm 0,36$ & $12,99 \pm 0,50$ & \\
\hline VICO & $-4,70 \pm 0,06$ & $10,95 \pm 0,04$ & $\mathbf{0 , 0 6} \pm \mathbf{0 , 1 3}$ & $-4,75 \pm 0,10 \mid$ & $10,98 \pm 0,04$ & $\mathbf{0 , 0 5} \pm \mathbf{0 , 1 1}$ & $-4,75 \pm 0,39$ & $\mathbf{1 0 , 9 7} \pm \mathbf{0 , 8 4}$ & $0,12 \pm 1,3$ \\
\hline
\end{tabular}
Fonte: Os autores (2021).

As velocidades obtidas pelo experimento Vel $\mathrm{TSR}_{\mathrm{TSR}}$ foi comparado com os demais. Os valores médios de discrepâncias entre os experimentos $\mathrm{Vel}_{\mathrm{TSR}}$ e $\mathrm{Vel}_{\mathrm{T}}$ são $-0,06 \mathrm{~mm} / \mathrm{ano}, 0,00 \mathrm{~mm} / \mathrm{ano}$ e $-0,15 \mathrm{~mm} / \mathrm{ano}$ nas componentes (e), (n) e (u), respectivamente. Já quando comparado o experimento Vel $\mathrm{TSR}_{\mathrm{T}}$ e Vel $\mathrm{l}_{\mathrm{TS}}$, os para as componentes (e), (n) e (u) são $-0,03 \mathrm{~mm} / \mathrm{ano},-0,02 \mathrm{~mm} / \mathrm{ano}$ e $-0,08 \mathrm{~mm} / \mathrm{ano}$, respectivamente. É possível perceber que não há uma variação significativa entre as velocidades obtidas pelos diferentes experimentos $\left(\mathrm{Vel}_{\mathrm{TSR}}\right.$ e $\mathrm{Vel}_{\mathrm{TS}}$ ), bem como, pelos experimentos $\mathrm{Vel}_{\mathrm{T}} \mathrm{e} \mathrm{Vel}_{\mathrm{TS}}$ nas componentes planimétricas, conforme pode ser observado a partir da Tabela 3.

Tabela 3 - Discrepância entre as velocidades a partir dos experimentos em (mm/ano).

\begin{tabular}{|c|c|c|c|c|c|c|c|c|c|}
\hline & \multicolumn{3}{|c|}{ VelTs $_{\text {- Vel }}$} & \multicolumn{3}{|c|}{ Vel $_{\text {TSR }}$ - Vel } & \multicolumn{3}{|c|}{ VelTSR - VelTS $_{\text {Th }}$} \\
\hline & $\Delta \mathrm{ve}$ & $\Delta \mathbf{v n}$ & $\Delta \mathbf{v u}$ & $\Delta \mathrm{ve}$ & $\Delta \mathbf{v n}$ & $\Delta \mathbf{v u}$ & $\Delta \mathbf{v e}$ & $\Delta \mathbf{v n}$ & $\Delta \mathbf{v u}$ \\
\hline Média & $-0,03$ & 0,02 & $-0,07$ & $-0,06$ & 0,00 & $-0,15$ & $-0,03$ & $-0,02$ & $-0,08$ \\
\hline Máximo & 0,04 & 0,31 & 0,31 & 0,32 & 0,14 & 0,30 & 0,36 & 0,08 & 0,33 \\
\hline Mínimo & $-0,14$ & $-0,13$ & $-0,61$ & $-0,23$ & $-0,18$ & $-0,76$ & $-0,20$ & $-0,29$ & $-0,81$ \\
\hline
\end{tabular}

Fonte: Os autores (2021).

Assim, pode-se inferir que o efeito da sazonalidade na determinação de velocidades planimétricas de estação GNSS na placa Sul-Americana (Brasil) não apresentou grandes variações quando processado no SARI.

Ressalta-se que o efeito da sazonalidade na componente (u) deve ser analisado com mais atenção, visto que o comportamento periódico desta componente é mais acentuado em relação às componentes (e) e (n). Isto confirma as afirmações de Berthier et al., (2019), que informa a importância de avaliar estimativas de velocidade da componente (u), visto que as águas superficiais (rios) não são incluídas nos modelos de carregamento hidrológico, considerando apenas a neve e a umidade do solo.

Algumas análises estatísticas dos índices espectrais (k) dos ruídos da lei de potência estimadas por 
MLE são apresentadas na Tabela 4.

Tabela 4 - Estatísticas básicas dos índices espectrais do experimento VTSR.

\begin{tabular}{ll|c|c|c|c}
\hline & \multicolumn{1}{c|}{ Componente } & $\mathbf{k}_{\text {médio }}$ & $\boldsymbol{\sigma}$ k-médio & Máximo & Mínimo \\
\hline \multirow{3}{*}{$\mathbf{V}_{\text {TSR }}$} & Este & $-0,90$ & 0,05 & $-0,60$ & $-1,17$ \\
& Norte & $-1,18$ & 0,03 & $-0,97$ & $-1,47$ \\
& $\mathbf{U p}$ & $-0,99$ & 0,05 & $-0,79$ & $-1,51$ \\
\hline
\end{tabular}

Fonte: Os autores (2021).

A partir da Tabela 4 é possível perceber, de acordo com os índices espectrais médios ( $\mathrm{k}_{\text {médio }}$ ), que os ruídos e as leis de potência presentes nas séries de coordenadas obtidas com GNSS são ruídos de cintilação (k $\cong-1$, flicker) e são semelhantes em todas as componentes. Logo, pode-se inferir que a área de estudos está sujeita a ruídos idênticos, já que as três componentes possuem a combinação de ruídos brancos combinados com ruídos de cintilação (ruídos da lei de potência).

Também é percebido que as precisões das coordenadas nos experimentos $\mathrm{Vel}_{\mathrm{T}}$ e $\mathrm{Vel}_{\mathrm{TS}}$ são mais otimistas quando comparados com experimento $\mathrm{Vel}_{\mathrm{TSR}}$, conforme apresentado na Tabela 5.

Tabela 5 - Precisões médias das velocidades de cada experimento em (mm/ano).

\begin{tabular}{|c|c|c|c|c|c|c|c|c|c|}
\hline & \multicolumn{3}{|c|}{ Vel $_{\mathbf{T}}$} & \multicolumn{3}{|c|}{ VelTs } & \multicolumn{3}{|c|}{ VelTSR } \\
\hline & $\sigma \mathrm{Ve}$ & $\sigma \mathrm{Vn}$ & $\sigma \mathrm{vu}$ & $\sigma \mathrm{Ve}$ & $\sigma \mathrm{Vn}$ & $\sigma \mathrm{vu}$ & $\sigma \mathrm{Ve}$ & $\sigma \mathrm{Vn}$ & $\sigma \mathrm{Vu}$ \\
\hline Média & 0,05 & 0,04 & 0,11 & 0,08 & 0,05 & 0,09 & 0,35 & 0,54 & 0,84 \\
\hline Máximo & 0,18 & 0,10 & 0,35 & 0,18 & 0,12 & 0,26 & 0,66 & 1,35 & 3,92 \\
\hline Mínimo & 0,03 & 0,02 & 0,05 & 0,03 & 0,02 & 0,04 & 0,18 & 0,24 & 0,37 \\
\hline
\end{tabular}

Fonte: Os autores (2021).

Ao realizar as análises das precisões entre os experimentos $\mathrm{Vel}_{\mathrm{T}} \mathrm{e} \mathrm{Vel}_{\mathrm{TS}}$ com o experimento Vel $\mathrm{l}_{\mathrm{TSR}}$, é possível perceber que os valores de precisão são, em média, 4, 10 e 7 vezes maiores nas componentes (e), (n) e (u), respectivamente. Ressalta-se que, de acordo com Klos, Bos e Bogusz (2018), qualquer sinal sazonal ou periodicidade residual não modelado e removido adequadamente, moverá a parte estocástica para um ruído muito mais correlacionado, fazendo com que as incertezas sejam superestimadas, ou seja, pode-se inferir que os valores de precisão das velocidades sejam superestimados quando comparado com as precisões obtidas no experimento que realiza análises de ruídos ( $\left.\mathrm{Vel}_{\mathrm{TSR}}\right)$.

Em seguida, foram comparadas as velocidades obtidas no experimento Vel $\mathrm{TSR}_{\mathrm{TSR}}$ com os valores da realização SIRGAS-CON (SIR17P01, época 2015). As discrepâncias entre os valores de velocidades obtidas entre o experimento $\mathrm{Vel}_{\mathrm{TSR}}$ e a realização SIRGAS-CON (VelsIRP01) são apresentadas na Figura 4. 
Figura 4 - Discrepância entre as velocidades obtidas no experimento $\mathrm{Vel}_{\mathrm{TSR}}$ com os valores obtidos na realização

SIRGAS-CON ( Vel $\left._{\text {SIRP01 }}\right)$, valores em (mm/ano).

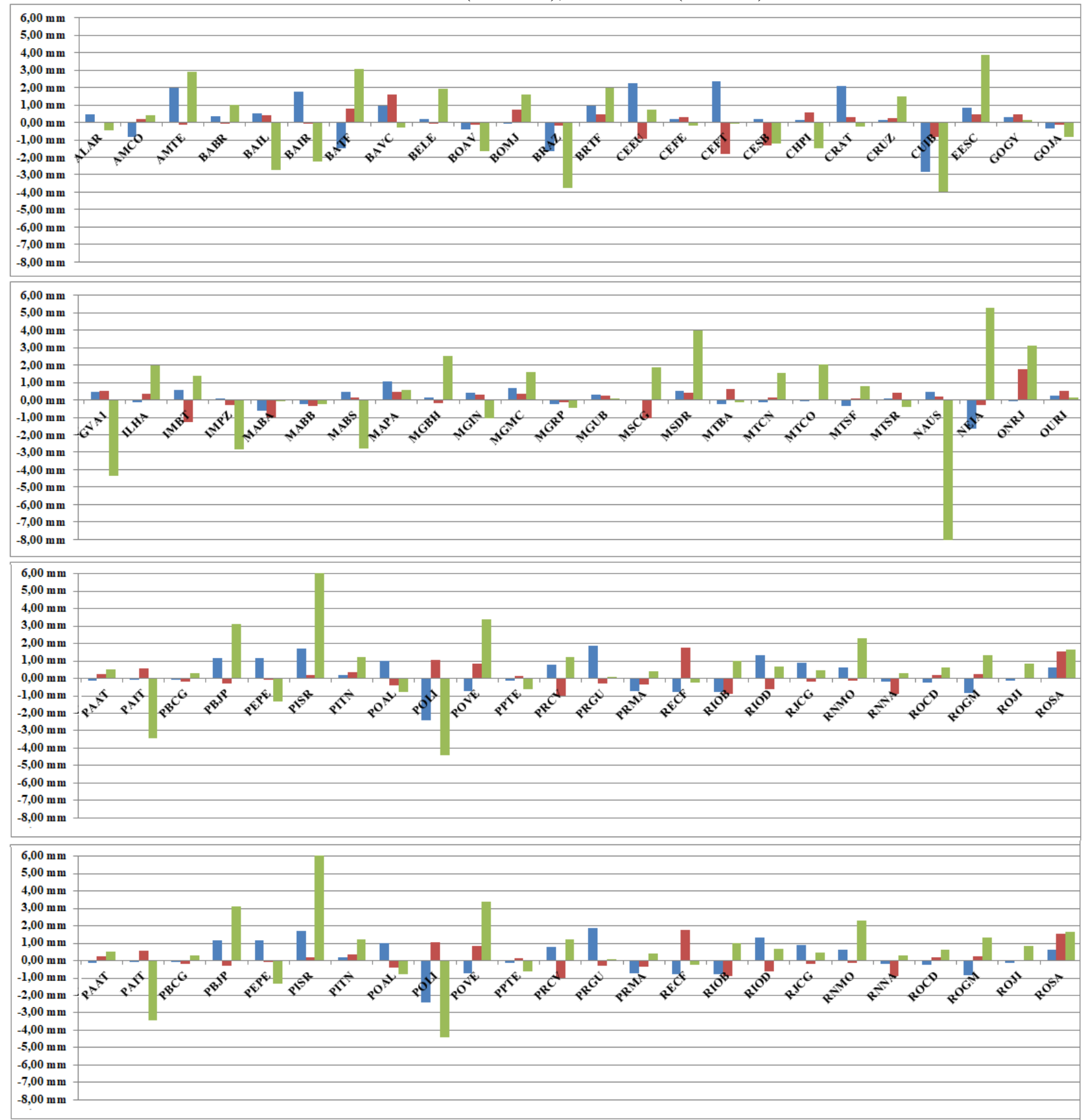

Fonte: Os autores (2021).

Os valores médios de discrepâncias absolutas obtidas a partir da Figura 4 nas componentes (e), (n) e (u) são 0,69 mm/ano, 0,50 mm/ano e 1,68 mm/ano, respectivamente. As discrepâncias máximas obtidas em cada componente foram 2,82 mm/ano, $2,37 \mathrm{~mm} /$ ano e 8,71 mm/ano, nas componentes (e), (n) e (u), respectivamente. Também é percebido que as discrepâncias são menores que $1 \mathrm{~mm}$ em $81 \%, 87 \%$ e $37 \%$ nas componentes (e), (n) e (u), respectivamente, evidenciando-se, assim, uma concordância nas componentes horizontais e um comportamento diferenciado da componente (u) quando se compara as velocidades disponibilizadas pela realização SIRGAS-CON (Vel SIRP01 $_{\text {) }}$ com os valores obtidos pelo experimento Vel $\mathrm{TSR}_{\text {TSR }}$

Para possibilitar uma melhor visualização das velocidades das estações analisadas nesta pesquisa, será apresentada a Figura 5, que corresponde aos vetores resultantes das velocidades planimétricas do experimento Vel $_{\text {TSR }}$ e VelsIRP01. 
Figura 5 - Vetores resultantes das velocidades planimétricas do experimento $\mathrm{Vel}_{\mathrm{TSR}}$ (vetores azuis) e Vel $\mathrm{l}_{\mathrm{SIRP} 01}$ (vetores

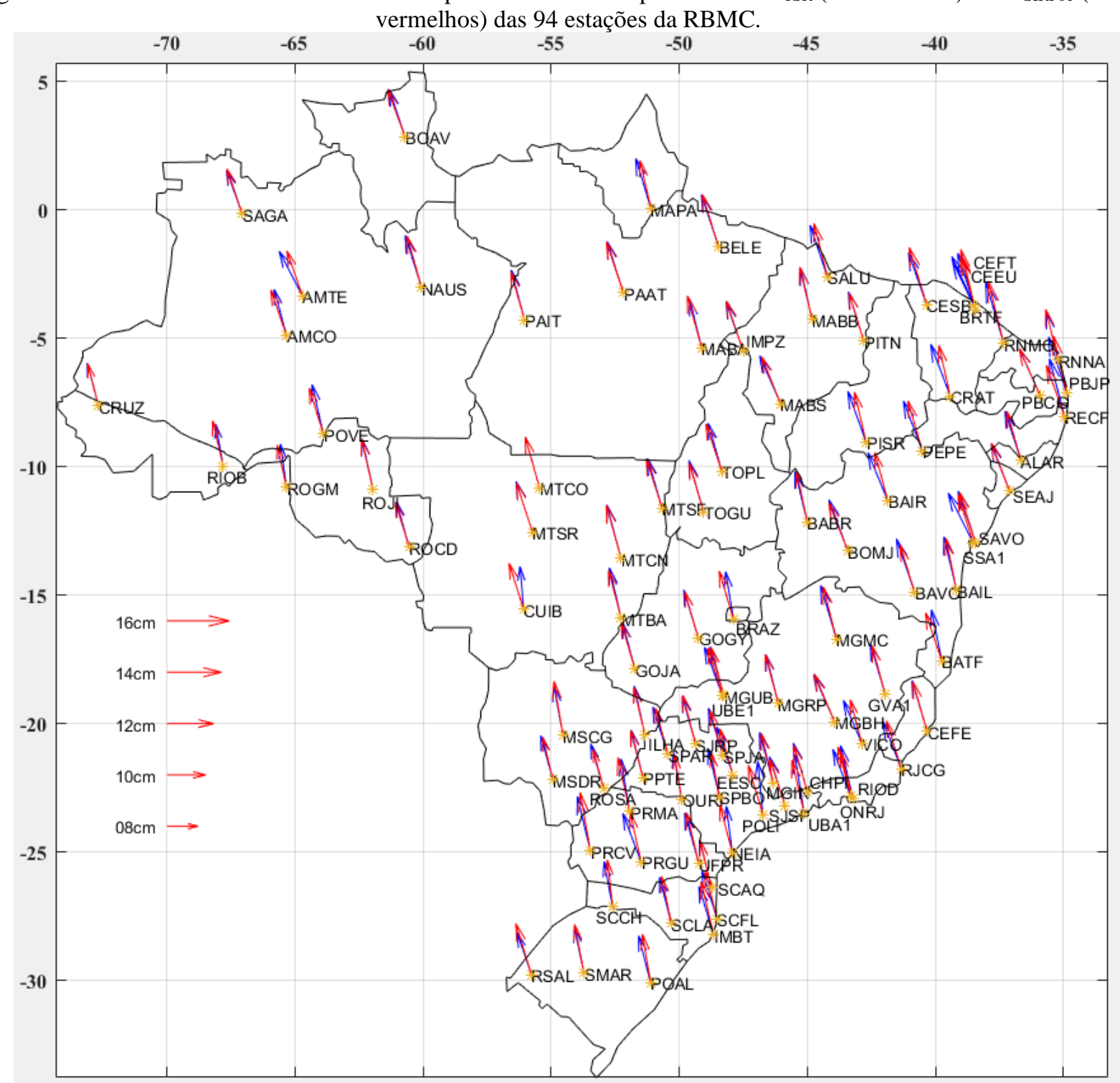

Fonte: Os autores (2021).

Já com relação à Figura 5, é possível perceber que as velocidades de algumas estações apresentam resultados com diferenças consideráveis entre os métodos de determinação de velocidade de estações, não sendo possível regionalizar os resultados encontrados. É importante acentuar que a os valores de VelsIRP01

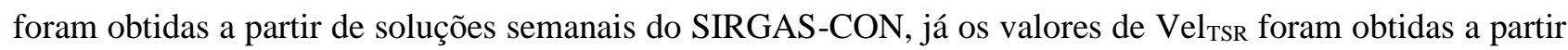
de posições diárias das estações.

\section{CONSIDERAÇÕES FINAIS E CONCLUSÕES}

Este trabalho apresenta técnicas de determinação de velocidades de estação GNSS para placa tectônica Sul-Americana a partir dos dados da RBMC, pós-processadas no IBGE-PPP e análises das séries temporais de coordenadas executada no software SARI.

Inicialmente, é possível afirmar que o software SARI apresenta-se como um software com grande potencial na realização de análises de séries temporais de coordenadas obtidas com uso do GNSS, por apresentar fidelidade em suas aplicações, uma vez que os ruídos que afetaram as séries temporais de coordenadas obtidas com GNSS, estudados nesta pesquisa, foram ruídos brancos e ruídos de cintilação (ruídos da lei de potência), o que confirma com os resultados de (WANG; HERRING, 2019; SANTAMARÍAGÓMEZ, 2019; BENOIST et al., 2020; HE et al., 2019; BOS et al., 2020). Isto mostra a eficiência do software SARI na determinação dos ruídos de lei de potência por meio da estimativa MLE. O software tem uma interface 
gráfica de fácil interação, e a entrada e saída de dados são realizadas de forma simples e eficientes. Os dados gráficos são interativos, sendo possível, e de maneira eficiente, a identificação e retirada de outliers das séries.

É possível perceber a estabilidade da placa tectônica Sul-Americana na porção analisada nesta pesquisa, visto que todas as componentes, analisadas separadamente, apresentam valores semelhantes. Estes dados reforçam os resultados obtidos em Sánchez e Drewes (2020), que afirmam que as únicas áreas estáveis da América Latina são o escudo das Guianas, Brasil e Atlântico. Outro fator importante identificado nesta pesquisa é com relação à análise da sazonalidade: pode-se afirmar que, quando a sazonalidade é negligenciada na determinação de velocidades planimétricas das estações da RBMC, as alterações não são significativas. $\mathrm{O}$ mesmo não ocorre nas componentes (u), que possuem comportamentos periódicos mais acentuados.

Por fim, é percebido que os ruídos que afetaram as séries temporais de coordenadas do GNSS, estudados nesta pesquisa, foram ruídos brancos e ruídos de cintilação (ruídos da lei de potência), o que confirma com os resultados de Wang e Herring (2019); Santamaría-Gómez (2019); Benoist et al. (2020); He et al. (2019); Bos et al. (2020). Também é possível perceber que os ruídos não afetaram nos valores das velocidades das estações, o que corrobora a afirmação de Klos et al. (2018), que informam que os ruídos só começam a se tornar mais importantes que os sinais periódicos para séries com mais de 9 anos. Nesta pesquisa foram analisados 7 anos de dados da determinação das velocidades das estações. Porém, foi notada uma depreciação significativa nas precisões das velocidades das estações quando comparada com os experimentos que não aplicaram as análises de ruídos. Contudo, conforme supracitado, qualquer sinal sazonal ou periodicidade residual não modelado e removido adequadamente, moverá a parte estocástica para um ruído muito mais correlacionado, fazendo com que as incertezas sejam superestimadas.

\section{Agradecimentos}

À Universidade Federal de Juiz de Fora pelo apoio concedido através do PROQUALI (Programa de Apoio à Qualificação). Aos colegas do Programa de Pós-Graduação em Engenharia Civil, em especial, ao Gabriel Diniz pela ajuda na obtenção dos dados da pesquisa.

\section{Contribuição dos Autores}

A conceptualização foi realizada por todos os autores, sendo o primeiro autor responsável pela curadoria dos dados, investigação, metodologia, validação, bem como a redação (minuta inicial, revisão e edição). Os demais autores (segundo e terceiro) foram responsáveis pela análise formal e supervisão do projeto.

\section{Conflitos de Interesse}

Os autores declaram que não há conflito de interesse.

\section{Referências}

ALINIA, H. S.; TIAMPO, K. F.; JAMES T. S. GPS coordinate time series measurements in Ontario and Quebec, Canada. Journal of Geodesy, v. 91, n. 6, p. 653-683, 2017. DOI. 10.1007/s00190-016-0987-5

ALVES, D. B. M.; SOUZA, E. M.; KANESHIRO, V. Y.; SOUZA, J. S. Análise de séries temporais de multicaminho em estações de monitoramento contínuo. Boletim de Ciências Geodésicas, v. 19 - p.353373, 2013. DOI. 10.1590/s1982-21702013000300001

BENOIST, C., COLliliEUX, X., REBISCHUNG, P., ALTAMIMI, Z., JAMET, O., MÉTIVIER, L., BEL, L. Accounting for spatiotemporal correlations of GNSS coordinate time series to estimate station velocities. Journal of Geodynamics, 2020. DOI. 10.1016/j.jog.2020.101693

BERTHIER, A., NICOLAS, J., VERDUN, J., BOY, J. P. MSSA Analysis Of Seasonal Loading-Induced Deformation From GNSS Time Series. In: 27th IUGG General Assembly $-8-18$ July 2019. Montreal, Canada. Poste... Montreal, Canada, 2019. 
BLEWITT, G.; KREEMER, C.; HAMMOND, W. C.; GAZEAUX, J. MIDAS robust trend estimator for accurate GPS station velocities without step detection. Journal of Geophysical Research: Solid Earth, v. 121, n. 3, p. 2054-2068, 2016. DOI. 10.1002/2015jb012552

BOCK, Y.; DIEGO M. Physical applications of GPS geodesy: a review. Reports on Progress in Physics, 2016. DOI. 10.1088/0034-4885/79/10/106801

BOGUSZ, J.; KLOS, A.; GRUSZCZYNSKA, M.; GRUSZCZYNSKI, M. Towards reliable velocities of permanent GNSS stations. Reports on Geodesy and Geoinformatics, v. 100, p.17-26, 2016. DOI. 10.1515/rgg-2016-0003

BOS, M. S.; FERNANDES, R. M. S.; WILLIAMS, S. D. P.; BASTOS, L. Fast error analysis of continuous GNSS observations with missing data. Journal of Geodesy, v. 87, n. 4, p. 351-360, 2013. DOI. 10.1007/s00190-012-0605-0

BOS, M. S., MONTILLET, J. P., WILLIAMS, S. D., FERNANDES, R. M. Introduction to Geodetic Time Series Analysis. In Geodetic Time Series Analysis in Earth Sciences. In: Geodetic Time Series Analysis in Earth Sciences. Springer, Cham, 2020. p. 29-52. DOI. 10.1007/978-3-030-21718-1

BOX, G. E. P.; JENKINS, G. M.; REINSEL, G. C. Time series analysis - Forecasting and control. Fourth Edition. Madison, Wisconsin: Ed. Wiley, 2008. DOI. 10.1002/9781118619193

CAÑADA, L. G. Análisis de series temporales en estaciones permanentes GPS. 2016. Tese de doutorado. Madrid: Universidad Complutense de Madrid, 2016.

CAPORALI, A. Average strain rate in the Italian crust inferred from a permanent GPS network-I. Statistical analysis of the time-series of permanent GPS stations. Geophysical Journal International, v. 155, n. 1, p. 241-253, 2003. DOI. 10.1046/j.1365-246x.2003.02034.x

CARVALHO, A. S. Implicações do emprego de diferentes modelos de velocidades e parâmetros da transformação de Helmert no posicionamento geodésico na placa sul-americana. 2015. Tese PósGraduação em Engenharia Civil da UFV. Viçosa-MG, 2015.

CHOUSIANITIS, K. GANAS, A; PAPANIKOLAOU, M, ARGYRAKIS, P; DRAKATOS, G; MAKROPOULOS, K. Time series analysis of the NOANET CGPS stations. Bulletin of the Geological Society of Greece, Chania, v. XLVII, 2013. DOI. 10.12681/bgsg.11078

COSTA, S. M. A. Estimativa do Campo de Velocidades a partir das Estações da RBMC. In: Instituto Brasileiro de Geografia e Estatística - IBGE, Departamento de Geodésia. Rio de Janeiro, 2001. Disponível em: <https://artigos.ibge.gov.br/artigos-home/geodesia/2004-2000/3100-estimativa-docampo-de-velocidades-a-partir-das-estacoes-da-rbmc>. Acesso em: 24 out. 2019.

DIDOVA, O.; GUNTER, B.; RIVA, R.; KLESS, R.; ROESE-KOERNER, L. An approach for estimating timevariable rates from geodetic time series. Jornal de Geodésia, 2016. DOI. 10.1007/s00190-016-0918-5

DREWES, H.; SÁNCHEZ, L. Velocity model for SIRGAS 2017: VEMOS2017. Technische Universitaet Muenchen, Deutsches Geodaetisches Forschungsinstitut (DGFI-TUM), IGS RNAAC SIRGAS, supplement to: SÁNCHEZ, L.; DREWES, H. Geodetic monitoring of the variable surface deformation in Latin America. International Association of Geodesy Symposia Series, v. 152, p. 01-12, 2020. DOI. 10.1007/1345_2020_91

EYER, L.; BARTHOLDI, P. Variable stars: which Nyquist frequency?. Astronomy and Astrophysics Supplement Series, v. 135, n. 1, p. 1-3, 1999. DOI. 10.1051/aas:1999102

GEIRSSON, H. Continuous GPS measurements in Iceland 1999 - 2002. 2003. Dissertação de mestrado em Ciência Geofísica. University of Iceland for the degree of Master of Science in Geophysics. Reykjavík, 2003.

HE, X.; MONTILLET, JEAN-PHILIPPE; FERNANDES, RUI; BOS, MACHIEL; YUF, KEGEN; HUA, XIANGHONG; JIANG, WEIPING. Review of current GPS methodologies for producing accurate time series and their error sources. Journal of Geodynamics, v. 106, p. 12-29, 2017. DOI. 10.1016/j.jog.2017.01.004 
HE, X., BOS, M. S., MONTILLET, J. P.; FERNANDES, R. M. S. Investigation of the noise properties at low frequencies in long GNSS time series. Journal of Geodesy, 93(9), 1271-1282, 2019. DOI. 10.1007/s00190-019-01244-y

INSTITUTO BRASILEIRO DE GEOGRAFIA E ESTATÍSTICA (IBGE). Manual do usuário aplicativo online IBGE-PPP. Rio de Janeiro: 2017. Brasil, 2017. Disponível em: <https://biblioteca.ibge.gov.br/visualizacao/livros/liv101677.pdf>. Acesso em: 10 jun. 2020.

KEITT, T. H. Coherent ecological dynamics induced by large-scale disturbance. Nature, v. 454, n. 7202, p. 331-334, 2008. DOI. 10.1038/nature06935

KLOS, A.; OLIVARES, G.; TEFERLE, F. N.; HUNEGNAW, A.; BOGUSZ, J. On the combined effect of periodic signals and colored noise on velocity uncertainties. GPS Solutions. v. 22, n. 1, p. 13, 2018. DOI. 10.1007/s10291-017-0674-x

KLOS, A; BOS, M. S.; BOGUSZ, J. Detecting time-varying seasonal signal in GPS position time series with different noise levels. GPS Solutions, v. 22, n. 1, p. 21, 2018. DOI: 10.1007/s10291-017-0686-6

LIMA, J. N. Análise do ruído das séries temporais GNSS. $3^{\circ}$ Encontro Nacional de Geodesia Aplicada. Lisboa, Portugal, 18 e 19 de Outubro de 2012.

LUNA, M. P.; STALLER, A.; TOULKERIDIS, T.; PARRA, H. Methodological approach for the estimation of a new velocity model for continental Ecuador. Open Geosciences, v. 9, n. 1, p. 719-734, 2017. DOI. 10.1515/geo-2017-0054

MAZZOTTI, S.; DEPREZ, A.; HENRION. E.; MASSON, C.; MASSON, F.; MENUT, J.; MÉTOIS, M.; NOCQUET,J.; ROLLAND, L.; SAKIC, P.; SOCQUET, A.; SANTAMARÍA-GÓMEZ, A.; VALTY, P.; VERGNOLE, M.; VERNANT, P. Comparative analysis of synthetic GNSS time series-Bias and precision of velocity estimations. Research Report, RESIF. 2020.

MORETTIN, P. A.; TOLOI, C. M. C. Análise de séries temporais. Vol. $2^{a}$ edição. São Paulo: Blucher, 2006.

NASCIMENTO, L. A. Influência da carga hidrológica na altitude geométrica a partir de análise de séries temporais estimadas no método PPP. 2016. 73f. Dissertação (mestrado em Engenharia Civil) da UVF. Viçosa, 2016.

NASCIMENTO, L. A.; DAL POZ, W. R.; SANTOS, A. D. P.; FREITAS, K. X. S. Geração automatizada de séries temporais de dados posicionais utilizando o IBGE-PPP. Boletim de Ciências Geodésicas, v. 23, no1, p.150-165, jan - mar, 2017. DOI. 10.1590/s1982-21702017000100010

NIKOLAIDIS, R. Observation of Geodetic and Seismic with the Global Positioning System. 2002. $190 f$. Tese de doutorado da University of California. San Diego, 2002.

PEREZ, J. A. S.; MONICO, J. F. G.; CHAVES, J. C. Velocity Field Estimation Using GPS Precise Point Positioning. The South American Plate Case, v. 2, n. 2, p. 90-99, 2003. DOI. 10.5081/jgps.2.2.90

PEREZ, J. A. S. Campo de velocidade para as estações da RBMC e do IGS localizadas na Plana SulAmericana: Estimadas a partir do processamento de dados GPS. 2002. 166f. Dissertação da Universidade Estadual Paulista. Presidente Prudente, 2002.

RAUCH, H. E.; TUNG, F.; STRIEBEL, C. T. Maximum likelihood estimates of linear dynamic systems. AIAA Journal, v. 3, n. 8, p. 1445-1450, 1965. DOI. 10.2514/3.3166

ROSA, G. P. S. Análise de séries temporais de coordenadas estimadas com GPS: uma proposta metodológica para eliminação de efeitos sazonais. 2008. 106f. Dissertação (mestrado em Ciências Cartográficas) da UNESP, Presidente Prudente. Presidente Prudente, de 2008.

SALAMANCA, G. E. D. Modelagem da deformação da crosta continental do Norte dos Andes através de inversão de dados sísmicos e geodésicos. 2016. 221f. Tese de Doutorado da Universidade de São Paulo. São Paulo, 2016.

SÁNCHEZ, L.; DREWES, H. Crustal deformation and surface kinematics after the earthquakes in Latin America. Journal of Geodynamics. v. 102, p. 1-23, 2016. DOI. 10.1016/j.jog.2016.06.005

SÁNCHEZ, L; DREWES, H. Geodetic monitoring of the variable surface deformation in Latin America. In: 
International Association of Geodesy Symposia. Springer, Berlin, Heidelberg, 2020. p. 01-12. DOI. 10.1007/1345_2020_91

SANTAMARÍA-GÓMEZ, Alvaro. SARI: interactive GNSS position time series analysis software. GPS Solutions, v. 23, n. 2, p. 52, 2019. DOI. 10.1007/s10291-019-0846-y

SANTAMARÍA-GÓMEZ, A., BOUIN, M. N., COLLILIEUX, X., WÖPPELMANN, G. Correlated errors in GPS position time series: Implications for velocity estimates. Journal of Geophysical Research: Solid Earth, v. 116, n. B1, 148-227, 2011. DOI. 10.1029/2010jb007701

SILVA, A. L.; COSTA, S. M. A. Análises e resultados do centro de processamento SIRGAS - IBGE. In: II Simpósio Brasileiro de Ciências Geodésicas e Tecnologias da Geoinformação, 8 a 11 de setembro de 2008. Recife-PE. Anais... Recife-PE, 2008.

SOUZA, W. O. Modelagem do movimento da placa tectônica sul-americana por meio de dados VLBI e GNSS. 2013. 177f. Dissertação (Mestrado em Ciências Geodésicas e Tecnologias da Geoinformação) da UFPE. Recife, 2013.

VONDRÁK, J. Problem of smoothing observational data II. Bull. Astron. Inst. Czechoslov. v. 28, n. 2, p. 84 - 89, 1977.

WANG, L; HERRING, T. Impact of estimating position offsets on the uncertainties of GNSS site velocity estimates. Journal of Geophysical Research: Solid Earth, 2019. DOI. 10.1029/2019jb017705

WILLIAMS, S.; BOCK, Y.; FANG, P.; JAMASON, P.; NIKOLAIDIS, R.M.; PRAWIRODIRDJO, L.; MILLER, M.; JOHNSON, D.J. Error analysis of continuous GPS position time series. J. Geophys. Res., v. 109, n. B18, B03412, 2004. DOI. 10.1029/2003jb002741

WILLIAMS, S. D. P. CATS: GPS coordinate time series analysis software. GPS solutions, v. 12, n. 2, p. 147 153, 2008. DOI. 10.1007/s10291-007-0086-4

ZAMORA, J. M. Desarrollo, implementación y análisis de un modelo cinemático local de velocidades para costa rica basado en la integración y homogenización de datos de estaciones GNSS continuas. 2014. 105 f. Tesis ( Doctor en Ciencias Físicas). Escuela Técnica Superior de Ingenieros em Topografía, Geodesia y Cartografía. Universidad Politécnica de Madri. Madri, 2014.

\section{Biografia do autor principal}

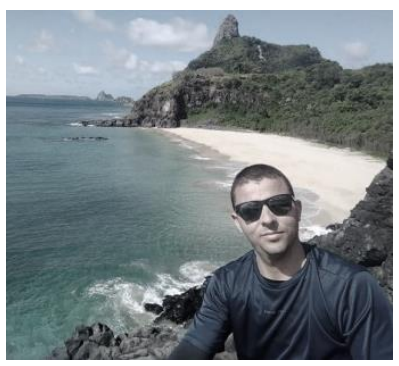

Marcony de Paulo Ramos, nascido em 05 de outubro de 1986 na cidade de Ipatinga - MG. Possui graduação em Engenharia de Agrimensura pela Universidade Federal de Viçosa - UFV; Mestre e doutorando em Engenharia Civil pela Universidade Federal de Viçosa, área de concentração em Informações Espaciais (linha de pesquisa em Geodésia). Foi professor da Universidade Federal de Juiz de Fora UFJF no Departamento de Transportes e Geodésia da Faculdade de Engenharia no período de 08/2016 a 08/2019. Atualmente é Professor na Universidade Federal da Bahia - UFBA no Departamento de Engenharia de Transportes e Geodésia.

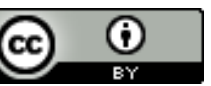

Esta obra está licenciada com uma Licença Creative Commons Atribuição 4.0 Internacional - CC BY. Esta licença permite que outros distribuam, remixem, adaptem e criem a partir do seu trabalho, mesmo para fins comerciais, desde que lhe atribuam o devido crédito pela criação original. 